\title{
Structural Brain Sex Differences in Parkinson’s Disease: A Voxel-Based Morphometry Study
}

\section{Abdullah A Alqarni*}

Department of Radiology and Medical Imaging, Prince Sattam Bin Abdulaziz University, Alkharj 11942, Saudi Arabia

\begin{abstract}
The aim of this study was to identify the brain's structural differences between males and females and how they relate to clinical manifestations of PD. Voxel-based morphometry (VBM) was used to detect the differences between both sexes in PD and in healthy controls (HC). Also, a Pearson correlation coefficient was used to correlate depression values to grey matter (GM) for PD and $\mathrm{HC}$ subjects and for males and females within each group. The VBM analysis results have shown large loss of cortical and subcortical regions in males compared to females in the PD and HC group. Moreover, multiple positive and negative correlations were found between depression and GM, and considered significant at $\mathrm{P}<0.05$. In addition, in $\mathrm{PD}$ group, depression scores were correlated positively to the right amygdala in females and positively in males. These results can provide insights for clinicians and researchers who are investigating PD structural differences in males and females and how they might influence PD clinical appearance, changes in motor and non-motor areas might explain the clinical manifestations.
\end{abstract}

Keywords: Parkinson's disease; VBM; Structural MRI; Grey Matter; Brain sexual differences; Brain dimorphism

\section{Introduction}

Parkinson's disease (PD) is one of the most common neurodegenerative and progressive diseases that is represented with motor and non-motor system deficiencies [1]. The onset of the disease is expected to be between 60 and 80 years of age on average [2,3]. In most cases, the disease is not linked to a specific cause; however, there are several known genes that can express forms of PD when mutated, and $5 \%$ to $10 \%$ of PD patients had been diagnosed with PD after one of these genes mutated [2,3]. Thus, there are two identified types of PD (i.e., familial and idiopathic). Familial PD can be autosomal dominant (AD) or autosomal recessive inheritance (AR). Parkinson's disease could result from the alteration of the genes' functional biochemistry, such as from oxidative stress, mitochondrial dysfunction, disturbance in protein quality control, and altered kinase activity that results in a range of neurological disorders [4,5] Furthermore, the effects of the disease, such as some of the clinical symptoms, age of onset, penetrance, severity, and disease progression, are thought to emerge in different ways based on the genetic variations, idiopathic (sporadic) interaction among genes, and environmental factors or dominance by single gene [5]. There are a few genes identified as single dominant genes that cause PD when mutated: SNCA-PARK1/PARK4 (alpha-synuclein protein), PRKN (parkin), PINK1, DJ-1, and LRRK2 [5].

Idiopathic (sporadic) PD is the most common version of PD, and it is the interest of this study. The disease is caused by hormonal, biochemical, environmental, and psychological factors, which have allowed investigators to explore those factors and provide different hypotheses to understand the complexity of this version [6]. In addition, other theories have been proposed by many investigators recently as to what might cause PD. According to Brown et al. [7], patients who are affected by inflammation in the brain due to stress caused by aging and environmental toxins might develop the causal effects of PD. Moreover, in other recent publications, the deterioration of the brain neurons caused by this continuous inflammation was found to cause neurological disorders $[8,9]$. The main pathological perspective is the loss of dopaminergic neurons in the substantia nigra pars compacta $(\mathrm{SNpc})$ that will lead to the course of the disease symptoms [1]. Furthermore, it is thought that PD symptoms might not be revealed until $60 \%$ of SNpc neurons are lost [6].
Motor and non-motor symptoms accompany the disease; motor symptoms are revealed when the basal ganglia deteriorate due to the lack of dopamine signals as a result of the neurons loss in SNpc; meanwhile, a few non-motor symptoms might be expressed before motor symptoms appear, such as cognitive decline, behavior or neuropsychiatric changes, and autonomic dysfunction [1]. Four symptoms classically recognize motor symptoms: resting tremor, rigidity, bradykinesia (slowness of movement), and postural instability; however, there are additional clinical features that could be classical motor symptoms, such as flexed posture (late in the disease) and freezing [10]. On the other hand, non-motor symptoms could have the same effects as motor symptoms on PD patients [10]. These non-motor features include autonomic dysfunction, such as orthostatic hypotension, sweating dysfunction, sphincter dysfunction, and erectile dysfunction [11-13], cognitive and neurobehavioral irregularities, such as dementia, depression, apathy, anxiety, and hallucination [14,15], and sleep disorders, usually exhibited by excessive sleepiness as well as rapid eye movement disorder $[10,16]$.

\section{Diagnosis of Parkinson's disease}

Diagnosis of PD is the most credible when there is a response to PD drugs (e.g., levodopa) [8,17]. Moreover, a reliable and validated biomarker that might help with revealing and diagnosing PD or its progression is currently absent [18]. Furthermore, medical imaging can only be used to discriminate PD from other neurological disorders with similar characteristics [17]. For instance, magnetic resonance imaging (MRI) might be useful in distinguishing PD from PSP, and essential tremors could be differentiated from PD utilizing singlephoton emission computed tomography imaging [17]. Clinically, there

*Corresponding author: Abdullah A. Alqarni, Department of Radiology and Medical Imaging, Prince Sattam Bin Abdulaziz University, Alkharj 11942, Saudi Arabia, Tel: 00966544441666; E-mail: abdullah.alqarni.89@gmail.com

Received August 03, 2017; Accepted September 27, 2017; Published October 12, 2017

Citation: Alqarni AA (2017) Structural Brain Sex Differences in Parkinson's Disease: A Voxel-Based Morphometry Study. J Neurol Disord 5: 364. doi:10.4172/23296895.1000364

Copyright: @ 2017 Alqarni AA. This is an open-access article distributed under the terms of the Creative Commons Attribution License, which permits unrestricted use, distribution, and reproduction in any medium, provided the original author and source are credited. 
are specific symptoms that might help clinicians distinguish PD from other neurological disorders; patients would have a better chance of being diagnosed with PD if they show micrographia (handwriting problems), which is linked to bradykinesia, a shuffling walk, and difficulty with special motor tasks, such as opening jars, bed natural turnovers, and standing from a chair-sitting position [19]. There are opposite symptoms that indicate that patients would not have PD, such as falls at onset, lack of reaction to levodopa, symmetrical resting tremor or less tremors at the early stage of the disease, accelerated rate of progression, and autonomic nervous system dysfunctions (e.g., orthostasis, constipation, dysphagia, urinary difficulties, and sexual dysfunction) [20]. However, UK Parkinson's Disease Society Brain Bank are well established criteria that are made to help clinicians to exclude symptoms that can complicate their decision [21].

\section{Treatment of Parkinson's disease (PD)}

There is no known cure for PD, but the symptoms could be controlled by using different types of pharmacological and nonpharmacological interventions [22]. The most known drug to reduce symptomatic clinical manifestations is Levodopa, it is thought that morbidity and mortality and clinical symptoms are decreased with the use of Levodopa [22,23].

\section{Sex Differences in Parkinson's disease (PD)}

Sex differences in PD were identified in many studies. The disease affects males more than females [24]. An exploratory study has shown that males have a different pattern of risk factors compared to females; occupational factors and the lifestyles of males, independently, are major risk factors [25]. Furthermore, motor symptoms are thought to be delayed in females compared to males $[26,27]$. According to Savica et al. [25], hormonal factors protect females, mainly the estrogen hormone. Other researchers supported this theory by emphasizing that a bilateral oophorectomy before the time when females stop having their period may naturally increase the risk of Parkinsonism due to reduced levels of estrogen $[28,29]$. Additionally, it is thought that estrogen could cause a lower striatal dopamine binding in males than females at the onset of the disease [27].

Genetically, sex chromosomes may include genes that are responsible for PD when mutated. Chromosome X has been associated with genetic susceptibility variants that might expound the sex factor differences in $\mathrm{PD}[25,30]$. For example, $\mathrm{X}$-linked dystonia parkinsonism (XDP) is one of the most aggressive genetic disorders that promotes Parkinsonism in adult onset, and the rate of males affected by this disease is significantly higher than females (male: female ratio 123:1) [30]. Dimorphism in the brain may play a role in developing PD or the way that the brain interacts with environmental factors [25]. Furthermore, multiple sex variations may appear during the disease or precede it [27]. Males are 1.5-2 times more susceptible to PD than females [31,32]. Moreover, on average, the onset of the disease is later in females by about 2.2 years when compared to males [33]. Furthermore, the Unified Parkinson's Disease Rating Scale (UPDRS) showed that males are more affected by motor symptoms than females, whereas dyskinesia was more prevalent in females than males $[34,35]$. In addition, with the increase of the disease's duration, more Parkinsonian symptoms affected males [36]. According to Haaxma et al. [27], estrogen status may play an important role before there are clinical manifestations of PD by delaying the onset of the disease in females. Additionally, the slow progression of the disease in females could be the reason for the predominant tremor that presents more in females than males [27]. Additionally, according to Martinez-Martin et al. [37], dyskinesia and depression symptoms related to $\mathrm{PD}$ are more frequent in females than males, while males have more often expressed rigidity and rapid eye movement compared to females.

\section{Normal brain dimorphism}

The interaction of biological and environmental factors influences the developing human brain, which is thought to result in brain dimorphism [38]. The size and weight of the human brain was investigated. On average, male brains are larger than female brains, with a $130 \mathrm{~cm}^{3}$ difference in the circumference [39]. Moreover, there is $10 \%$ increase in the size of male brains, including an increase in weight by $11 \%$ to $12 \%$ [40]. Furthermore, it is presumed that height plays a role in increasing the weight of the brain due to more neurons being required to control bigger bodies $[40,41]$. Males and females have brain regional differences regarding the volume of the brain cortex; frontal and medial paralimbic cortices are larger in volume when correlated to cerebrum size. Males have larger front medial cortex and amygdala and hypothalamus volumes when correlated to cerebrum size [40]. Sex steroids have a major impact on developmental studies because of their role in changing the structure of the homologous structures in the brain $[40,42]$. Sexual brain dimorphism was investigated in a recent metaanalysis study that included 126 studies for overall volume analysis and 24 studies that had performed voxel-based morphometry for regional volume and density meta-analysis [43]. In Ruigrok et al. [43], a descriptive overview of the brain's overall volume was made for the sexual brain dimorphism based on age categories. In addition, the data have been divided into six categories: infants ( 0 to 1$)$, early childhood (2 to 6), late childhood ( 7 to 17 ), mature (18 to 59 ), senior (over 60 ), and lifespan (all ages) [43]. However, underrepresentation in some of these categories (infant and early childhood) has been noted in the meta-analysis, while the most well-presented category was the mature category [43]. Therefore, the mature category might have affected the results of this meta-analysis due to the large sample of this category [43]. An overall increase in the average of male brain volume compared to female brain volume was found: intracranial volume (ICV; $12 \%$ ), total brain volume (TBV; $11 \%)$, cerebrum $\mathrm{Cb}(10 \%), \mathrm{GM}(9 \%)$, WM (13\%), cerebrospinal fluid (CSF; 11.5\%), and cerebellum (Cbl; 9\%) [43].

In the regional meta-analysis, a coordinate-based meta-analysis was performed on GM for regional volume and density [43]. Furthermore, the percentage of male brains included in this process was slightly higher than females by $3 \%$ [43]. Regarding regional volume and density differences between both sexes, males presented with an increased volume in bilateral limbic areas and left posterior cingulate gyrus and increased densities in the left limbic regions, while females presented with an increased volume in the language-related areas and some limbic regions in the right insula and anterior cingulate gyrus cortices [43]. Particularly, on average, the left amygdala, hippocampus, insular cortex, and putamen had more volume and density; the Right VI lobe of the cerebellum and the left claustrum had more density; bilateral anterior Para hippocampal gyri, posterior cingulate gyri, precuneus, temporal poles, and cerebellum areas in the left posterior and anterior cingulate gyri and in right amygdala, hippocampus, and putamen had larger volumes in male brains [43]. On the other hand, the left frontal pole had more density on average in female brains, as well as having increased volume in the right frontal pole, inferior, and middle frontal gyrus, pars triangularis, planum temporal or parietal operculum, anterior cingulate gyrus, insular cortex, and Heschl's gyrus, bilateral thalami and precuneus, the left Para hippocampal gyrus, and lateral occipital cortex, on average, in female brains [43].

\section{Depression in Parkinson's disease (PD)}

Depression is one of the symptoms that manifests in females 
in earlier stage than males [44]. Depression has been theoretically linked to the amygdala because the amygdala plays an important role in emotional memory [45]. It is thought that females exhibit higher levels of depression more than males at the onset of PD [46]. Amygdala volume has been investigated using structural neuroimaging to identify whether there are any volume differences between depressed and nondepressed subjects or not [45]. However, inconsistent results had been reported in studies that had investigated the volume of the amygdala; in these studies, the volume of the amygdala was smaller in depressed subjects and larger in other studies compared to subjects with no depression. Moreover, no differences were found in the volume of the amygdala between the two groups in other studies [47-54]. According to the meta-analysis conducted by Hamilton et al. [45], depression medications could increase and protect the amygdala by increasing its volume, whereas untreated depression may lead to a volume decrease of the amygdala.

\section{Study aims}

This study has the aim of identifying the major areas of the brain that are mainly affected by the disease, providing a reference for the future investigation of PD. Thus, a large sample of male and female subjects and healthy controls (HC), using whole brain voxel-based morphometric analysis on MRI images, were used to accomplish the aims of the study. Moreover, this study aims to illustrate sex dimorphism that result due to PD impact on both sexes. In addition, previous literature had suggested that PD-related depression is more prevalent in females; thus, a correlation between the results of the depression test and the findings from the VBM analysis will be performed to identify what areas were associated with depression scores.

\section{Hypotheses}

Primarily, sexual brain dimorphism is recognized in male and female brains due to genetical, hormonal, behavioral, and environmental factors; thus, the volume and intensity would be different in many regions in the brain. It is hypothesized that the symptoms of PD appear differently due to the impact of atrophy caused by PD on the brain. The major atrophied regions will be expressed differently in PD because of sexual brain dimorphism and the results of the VBM analysis will show different dimorphism of HC. Secondarily, it is hypothesized that PDrelated depression affects both males and females equally, however, the correlation test will indicate different relations of depression and GM areas in males and females.

\section{Materials and Methods}

\section{Subjects}

All clinical and MRI image data were obtained from the Parkinson's Progression Markers Initiative (PPMI) data base [55]. The PPMI project is an international, multicenter study that is an observational and comprehensive and used for identifying better interpretations of PD etiology, biomarkers for PD progression, and improving the medicinal trials for PD drugs [56]. The PPMI project has set an inclusion and exclusion criteria for PD and control subjects. The inclusion criteria for PD subjects were, firstly, having at least two motor symptoms from the known Parkinson's motor symptoms (resting tremor, bradykinesia, and rigidity). Secondly, from a baseline, the PD patients must be Stage 1 or 2 according to the Hoehn and Yahr test. Thirdly, a deficiency in dopamine transportation function screened by SPECT must be present. Fourthly, PD medications were not needed during at least 6 months from the baseline. Lastly, participants were not included if they were younger than 30 years of age. The exclusion criteria for PD subjects included patients who were consuming PD medications at the baseline or within 2 months after the baseline and if they had used any experimental devices or drugs 60 days before the baseline [57]. Healthy controls (HC) in the PPMI study were included if they were at the age of 30 or over. The exclusion criteria for HC subjects were, firstly, being clinically diagnosed of a neurological disorder whether it was recent or active. Secondly, if one of the family members (parent, sibling, or child) of the volunteer was a PD patient, the control was included [57]. A total of 572 subjects were included based on their brain scanning methodology. For all 572 subjects, structural T1 images were used from MRI scanners located at different centers around the world. However, nine subjects were excluded during the data analysis due to quality and software misdetection issues. The remaining 563 subjects were divided into two groups; HC and PD. The HC group contained 176 subjects, whereas the PD group included 387 subjects. Moreover, each group was subcategorized into male and female categories in order to detect the gender differences in the brain. The HC male subgroup involved 112 subjects, while the female subgroup involved 64 subjects. The PD male subgroup contained 247 subjects, whereas the female subgroup contained 140 subjects.

\section{Clinical assessment}

There are many clinical and neuropsychological tests that were performed on the subjects to diagnose and assess them in the PPMI project. Demographic data at the project's baseline were examined and included gender, age, number of relatives with $\mathrm{PD}$, duration of the disease, Hoehn and Yahr test score, Movement Disorder SocietyUnified Parkinson's Disease Rating Scale (MDS-UPDRS) test total score, Modified Schwab and England Activities of Daily Living test scores (Modified Schwab \& England ADL), Montreal Cognitive Assessment (MoCA), Scales for Outcomes in Parkinson's DiseaseAutonomic scores (COPA-AUT), and the University of Pennsylvania Smell Identification Test (UPSIT). In addition, depression and its drugs were examined. In this study, the Geriatric Depression Scale (GDS) and Concomitant Medications records were analyzed to test the link between depression and the subjects based on gender.

\section{Data analysis}

A whole brain analysis for the structural data is essential for examining the grey matter changes between PD and HC, as well as among the subgroups. In the present study, FSL-VBM was utilized to execute the pre-processing of the structural data (http://fsl.fmrib.ox.ac. uk/fsl/fslwiki/FSLVBM) [58]. The instructions and commands at the FSL-VBM website (http://fsl.fmrib.ox.ac.uk/fsl/fslwiki/FSLVBM) were followed to achieve an optimized protocol [59] while using FSL tools [60]. The data analysis was performed as follows: First, a directory was created and specified to contain all of the structural images; each group's images were renamed in accordance to their categorical status (HC, PD). Moreover, a template list, which is a text file that contains the images' titles, was created in the same directory so that the software could process the data accordingly. Second, an extraction of the brain was performed using the brain extraction tool (BET) command line, which helps exclude the neck from the images and preventing any confusion in this pre-processing step [61]. The results of this step needed to be checked; two hundred and thirty-one subjects were required to be re-processed because the BET did not work for cutting the skull, even when several thresholds were applied. To solve this issue, Free Surfer software was employed for cutting the skull. Basically, it extracts the brain by stripping the image from other non-brain tissues using a hybrid watershed or surface deformation procedure [62]. By the end of the second step, five subjects' images were excluded because they had not been brain-extracted due to software errors. 
Third, all extracted brains were placed in a stereotactic space; then, a full automated process was utilized to separate grey matter, white matter, and cerebrospinal fluid [59]. GM images were concatenated and averaged after the affine-registration to the GM ICBM-152 template. A first pass was achieved after flipping the averaged results along the $\mathrm{x}$-axis, followed by re-averaging of the two mirror images. A concatenated $4 \mathrm{D}$ image that holds all the GM images of the subjects was then produced by non-linear registration, which applies another registration of the GM images from the template list to the generated GM template. The resulting image was averaged and flipped along the $\mathrm{x}$-axis. In order to create the symmetric study-specific template, both mirror images were then automatically averaged. The resolution of the final template was a 2 $\times 2 \times 2 \mathrm{~mm}^{3}$ in standard space. Subsequently, the 4D image was checked for any unregistered GM images, and as a result, four subjects were excluded. Fourth, another concatenated 4D image was generated using non-linear registration to register the GM images to the study-specific template, and this image was automatically placed in the statistics directory so that the last step of the analysis could be performed. However, GM regional changes could occur because of the previous process; therefore, another automated processing was integrated to correct the volumetric changes (modulation), which maintains each voxel's volume of GM [59]. Then, it was confined into the previous GM $4 \mathrm{D}$ image. Smoothing with a range of Gaussian kernels has been applied to the image that was normalized, segmented, and modulated. For this study, five GM 4D images that were normalized, segmented, smoothed, and modulated were produced for each of the group comparisons (HC vs. PD, females of $\mathrm{HC}$ vs. females of PD, males of $\mathrm{HC}$ vs. males of PD, males of $\mathrm{HC}$ vs. females of $\mathrm{HC}$, males of PD vs. females of PD), the 5 GM 4D images were derived from the GM 4D image that contain all the GM data of the subjects and for time limits this step was done using MATLAB code [63]. The final step was preparing the $4 \mathrm{D}$ images for the automated statistic maps process. Furthermore, voxel wise statistical maps were fully deducted using randomization methods that use the standard general linear model (GLM) design to model and inference them [64] (Figure 1).

\section{Statistical analysis}

Comparison was set within FSL general linear model for PD and HC group and for comparisons between males and females in PD and HC subgroups, age was used as covariate. A two-sampled student $t$ test was performed on the demographic data (age, sex, disease duration, MDS-UPDRS, Modified Schwab and England ADL, UPSIT, MoCA, COPA-AUT, GDS) of the PPMI subjects (All HC vs. All PD, PD male vs PD female, HC male vs. HC females) at baseline, the test was undertaken to evaluate the state of subjects at baseline. To evaluate the relationship between depression and GM changes in males and females in PD and HC categories, Pearson correlation was used. A two-tailed $\mathrm{P}<0.05$ was considered statistically significant. MATLAB code was created to perform Pearson correlation [63]. A brain map was generated containing the significant clusters only. In addition, a chi-square test for categorical variables was performed to identify the sex differences of the study.

\section{Results}

\section{Demographic characteristics}

The significance level was set at $\mathrm{P}<0.05$ for the statistical analysis of the demographic characteristics as a baseline. For age, there were no significant differences when comparing all $\mathrm{HC}$ with all $\mathrm{PD}$, between $\mathrm{PD}$ males and females, and in HC males and females. Moreover, the chisquare results showed no significant effect regarding sex differences.

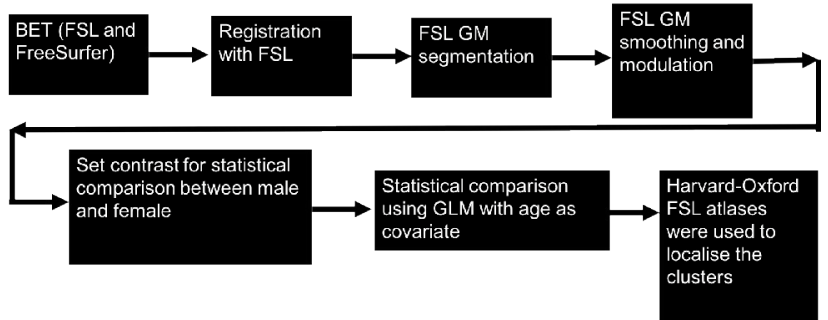

Figure 1: Steps that were undertaken to perform the data analysis for the set of data.

Furthermore, no differences in the disease duration were considered significant between PD males and females. The total score of MDSUPDRS the test was analyzed at $\mathrm{P}<0.05$, and there was a significant difference in all PD subjects compared to HC subjects $(\mathrm{P}<0.00001)$. However, there were no considerable variations between both sexes within PD and $\mathrm{HC}$ categories. In the PD group, a significant difference was not found between males and females for the Hoehn and Yahr test. PD patients' daily life activities were tested with the Modified Schwab and England ADL examination; in comparison, no significant differences between males and females were found. The University of Pennsylvania Smell Identification Test (UPSIT) was used to test smelling sense and was significantly higher in all PD subjects than in HC $(\mathrm{P}<0.00001)$. Moreover, statistically, the score was considerably higher in males than females in the $\mathrm{PD}$ group $(\mathrm{P}<0.000406)$, while no significance was detected in HC group. Also, all PD subjects had showed significantly higher cognitive defects than $\mathrm{HC}$ subjects $(\mathrm{P}<0.00001)$. In the PD group, males were affected more than females by cognitive deficiency $(\mathrm{P}<0.011593)$, while in the $\mathrm{HC}$ group, no significant differences were detected. Furthermore, in the Scales for Outcomes in Parkinson's Disease-Autonomic examination (COPA-AUT), PD subjects had a higher level of differences than HC subjects $(\mathrm{P}<0.00001)$. Moreover, males in the HC group showed significant impairment of the autonomic functions compared to females $(\mathrm{P}<0.033041)$, whereas no significant variations were found between both sexes in PD. For depression comparison, HC males showed a higher depression significance level $(\mathrm{P}<0.00015)$, whereas no significance was detected between PD and HC subjects or within the PD group between both sexes (Table 1).

\section{VBM results}

As previously descried, the 563 subjects of this study were divided into five groups: 1) $387 \mathrm{PD}$ subjects versus $176 \mathrm{HC}$ subjects; 2) $64 \mathrm{HC}$ females versus 140 females with PD; 3) $112 \mathrm{HC}$ males versus 247 males with $\mathrm{PD}$; 4) $112 \mathrm{HC}$ males versus $64 \mathrm{HC}$ females; and 5) 247 males with PD versus 140 females with PD. Moreover, the cluster-based correction for all resulted images was at $\mathrm{t}>3$. Statistical contrast was set within FSL general linear model for comparison.

PD subjects versus HC subjects: The results from the VBM showed a significant loss of brain tissue in PD subjects compared to HC subjects, and no loss was found in HC. The main tissue loss was focused in two large clusters; the first one indicates a significant loss in the right amygdala $(t=4.67$, MNI coordinates: $30,2,-22)$, the right planum polare ( $\mathrm{t}=3.25$, MNI coordinates: $46,-6,-10)$, the right temporal pole $(\mathrm{t}=3.329$, MNI coordinates: $24,8,-28)$, and the right Para hippocampal gyrus ( $\mathrm{t}=4.15$, MNI coordinates: $28,2,-20)$, whereas the second cluster includes the right putamen $(\mathrm{t}=3.62$, MNI coordinates: $26,8,10)$, the right insular cortex ( $\mathrm{t}=3.55$, MNI coordinates: $34,8,8)$, the right central opercular cortex $(t=3.45$, MNI coordinates: $38,8,10)$, and the right inferior frontal gyrus (pars opercularis; $\mathrm{t}=3.67$, MNI coordinates: 50 , 8,12 ) (Figure 2). 
Citation: Alqarni AA (2017) Structural Brain Sex Differences in Parkinson's Disease: A Voxel-Based Morphometry Study. J Neurol Disord 5: 364 doi:10.4172/2329-6895.1000364

Page 5 of 14

\begin{tabular}{|c|c|c|c|c|c|c|}
\hline \multirow{2}{*}{ Demographic Characteristics } & Total Subjects & \multicolumn{2}{|c|}{ PD } & \multicolumn{2}{|c|}{ HC } & P Value \\
\cline { 2 - 7 } & PD/HC & Males & Females & Males & Females & p<0.05 \\
\hline Age (years) & $62.17 \pm 9.8 / 60.5 \pm 9.8$ & $62.7 \pm 9.8$ & $61.2 \pm 9.7$ & $61.6 \pm 11.34$ & $58.7 \pm 11.5$ & - \\
\hline Sex & $387 / 167$ & 247 & 140 & 112 & 64 & - \\
\hline Disease Duration (months) & $6.6 \pm 6.6 /-$ & $6.5 \pm 6.24$ & $6.8 \pm 7.3$ & - & - & - \\
\hline MDS-UPDRS & $31.9 \pm 12.9 / 4.5 \pm 2.9$ & $32.22 \pm 12.9$ & $31.4 \pm 12.9$ & $4.16 \pm 3.48$ & $5.14 \pm 4.9$ & PD vs HC: $<0.00001$ \\
\hline Hoehn and Yahr stage & $1.5 \pm 0.5 /-$ & $1.5 \pm 0.5$ & $1.5 \pm 0.5$ & - & - & - \\
\hline Modified Schwab and England ADL & $93 \pm 5.8 /-$ & $92.8 \pm 5.4$ & $93.5 \pm 6.4$ & - & - & - \\
\hline UPSIT & $22.5 \pm 8.3 / 34.11 \pm 4.6$ & $21.3 \pm 8.2$ & $24.48 \pm 8.26$ & $33.7 \pm 5$ & $34.9 \pm 36$ & $\begin{array}{c}\text { PD vs HC: }<0.00001 \\
\text { PDM vs PDF=0.000406 }\end{array}$ \\
\hline MoCA Score & $27 \pm 2.29 / 28.2 \pm 1$ & $26.9 \pm 2.32$ & $27.5 \pm 2.2$ & $28.11 \pm 1.11$ & $28.4 \pm 1$ & $\begin{array}{c}\text { PD vs HC<0.00001 } \\
\text { PDM vs PDF=0.011593 }\end{array}$ \\
\hline COPA-AUT & $9.35 \pm 5.8 / 5.6 \pm 3.7$ & $9.36 \pm 6$ & $9.35 \pm 5.5$ & $5.28 \pm 3.13$ & $6.5 \pm 3.69$ & $\begin{array}{c}P D \text { vs HC<0.00001 } \\
\text { HCM vs HCF=0.033041 }\end{array}$ \\
\hline Education (years) & $15.5 \pm 3 / 16 \pm 2.9$ & $15.7 \pm 2.9$ & $15.23 \pm 3.14$ & $16.35 \pm 3$ & $15.48 \pm 2.8$ & - \\
\hline GDS & $5.4 \pm 1.5 / 4.5 \pm 2.18$ & $5.4 \pm 1.5$ & $5.3 \pm 1.37$ & $5 \pm 1.5$ & $5.1 \pm 1.3$ & HCM vs HCF=0.00015 \\
\hline
\end{tabular}

Table 1: Demographic characteristics of PD and HC subgroups and the results of the statistics tests (t-test, chi-square).

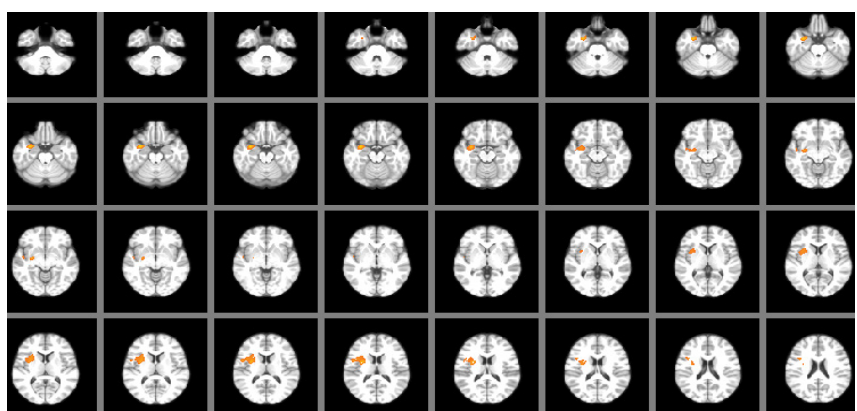

Figure 2: Identified clusters in the VBM comparison between $\mathrm{PD}<\mathrm{HC}$ subjects. Increased loss of GM identified by increasing warmth of colour. (Yellow is warmer.)

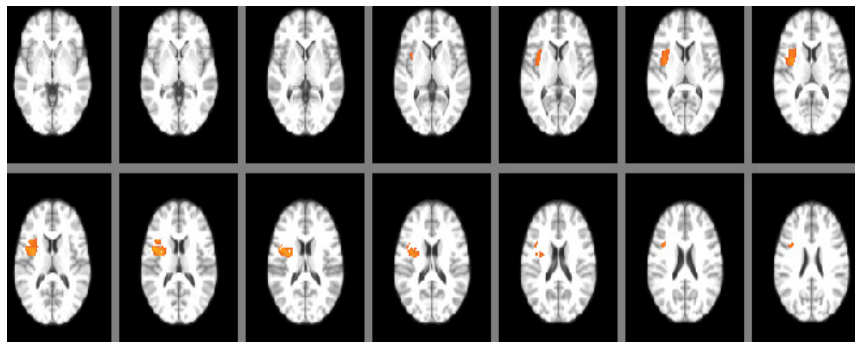

Figure 3: Identified clusters in the VBM comparison between PD females $<\mathrm{HC}$ females. Increased loss of GM identified by increasing warmth of colour in PD. (Yellow is warmer.)

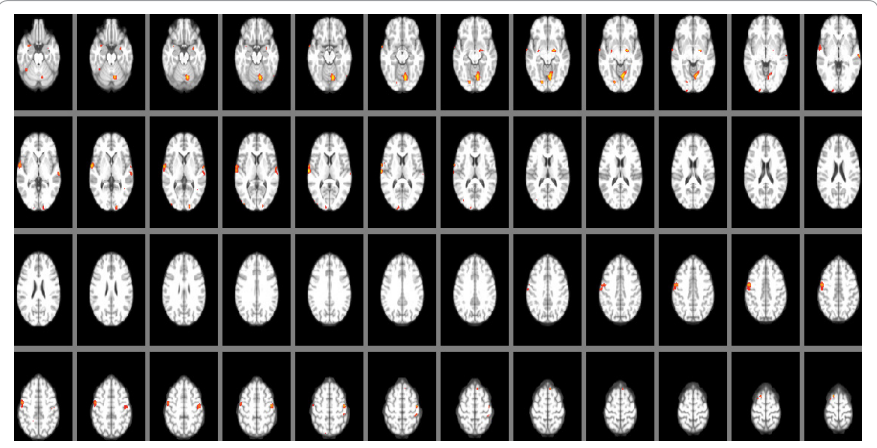

Figure 4: Identified clusters in the VBM comparison between PD males $<\mathrm{HC}$ males. Increased loss of GMin males with PD was identified by increasing the warmth of colour after the adjustment of threshold was moved from three to four $(\mathrm{t}=3-4)$. (Yellow is warmer.)

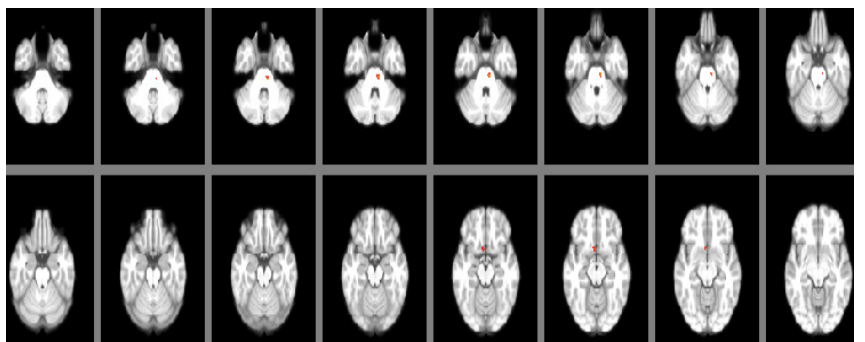

Figure 5: Identified clusters in the VBM comparison between $\mathrm{HC}$ males < males with PD. Increased loss of GM was identified in $\mathrm{HC}$ males by increasing the warmth of colour after adjusting the threshold from three to four $(t=3-4)$. (Yellow is warmer.)

HC females versus females with PD: In the comparison between female brains in PD and HC, brain tissue loss was significant in the right side of the brain only. Furthermore, the loss was detected in females with PD only in the right insula ( $\mathrm{t}=4.2$, MNI coordinates: 34,0 , 12), the right centeral opercular cortex $(t=4.134$, MNI coordinates: 40 , 2,14 ), and the right inferior frontal gyrus (pars opercularis; $t=3.6, \mathrm{MNI}$ coordinates: $36,10,24$ ) (Figure 3).

HC males versus males with PD: No differences were found between males in both categories at the threshold given in this study for all comparisons. However, when increasing the threshold in the FSL view window and restricting it between three and four $(t=3-4)$ for the uncorrected images, it shows areas that were affected in PD males. Areas that displayed this were the left VI of the cerebellum ( $t=3.8$, MNI coordinates: $-14,-68,-14)$, the lingual gyrus $(t=3.9$, MNI coordinates: $-14,-64,-8)$, the left putamen ( $t=3.2$, MNI coordinates: $-28,-10,-8)$, the precentral gyrus ( $\mathrm{t}=3.7$, MNI coordinates: $54,-4,44)$, and the planum polare ( $t=3.7$, MNI coordinates: $60,0,2)$ (Figure 4 ). On the other hand, males in the HC group presented with a loss of tissue in the uncorrected image, with the same previous restriction used. HC males showed a loss in the subcallosal cortex ( $t=3.306$, MNI coordinates: $4,12,-14)$ and brain-stem ( $\mathrm{t}=3.5$, MNI coordinates: $-10,-26,-34)$ (Figure 5).

HC males versus $\mathrm{HC}$ females: Loss of GM was significant in $\mathrm{HC}$ males compared to HC females; multiple clusters across the brain regions were detected in the VBM analysis. The loss of brain tissue in males was significant in the brain stem ( $\mathrm{t}=3.5 \mathrm{MNI}$ coordinates: $-2,-34$, $-40)$, the frontal medial cortex $(t=5.17$, MNI coordinates: $0,46,-20)$, the sub-callosal cortex ( $\mathrm{t}=3.27$, MNI coordinates: $0,26,-20)$, the right frontal pole $(\mathrm{t}=4.8$, MNI coordinates: $24,38,-20)$, the bilateral frontal orbital cortices (right: $\mathrm{t}=3.9$ MNI coordinates: 14,22 , -18 ; left: $\mathrm{t}=4.045$, 
Citation: Alqarni AA (2017) Structural Brain Sex Differences in Parkinson's Disease: A Voxel-Based Morphometry Study. J Neurol Disord 5: 364 doi:10.4172/2329-6895.1000364

MNI coordinates: $-20,22,-18$ ), the bilateral hippocampus (right: $\mathrm{t}=4.36$, MNI coordinates: $28,-32,-10$; left: $\mathrm{t}=5.17$, MNI coordinates: $-28,-30,-10)$, the left superior temporal gyrus $(t=3.6$, MNI coordinates: $-54,-36,2$ ), the bilateral middle temporal gyrus (right: $t=3.5$, MNI coordinates: $48,-42,8$; left: $\mathrm{t}=3.9$, MNI coordinates: $-54,-36,-2)$, the bilateral planum polare (right: $\mathrm{t}=3.7$, MNI coordinates: $42,-18$, -2 ; left: $\mathrm{t}=3.5$ MNI coordinates: $-44,-18,-2$ ), the bilateral thalamus (right: $\mathrm{t}=4.8$, MNI coordinates: $6,-6,6$; left: $t=4.19$, MNI coordinates: $-10,-18,16$ ), the bilateral insular cortices (right: $\mathrm{t}=4.21$, MNI coordinates: $38,-6,12$; left: $\mathrm{t}=4.42$, MNI coordinates: $-38,-6,10)$, the bilateral supramarginal gyrus (right: $\mathrm{t}=-38,-40,40$; left: $\mathrm{t}=5.33$, MNI coordinate: $42,-38,40$ ), the bilateral superior parietal lobule (right $\mathrm{t}=6.101$, MNI coordinates: 36, -42 , 44; left: $\mathrm{t}=5.7$, MNI coordinates: $-36,-42,44)$, the bilateral cingulate gyrus (right: $\mathrm{t}=4.8$, MNI coordinates: $4,36,16$; left: $\mathrm{t}=4.6$, MNI coordinates: $-2,36,12$ ), and the left juxta positional lobule cortex $(t=3.26$, MNI coordinates: $-4,0,52)$ (Figure 6). Furthermore, HC females showed no loss of brain tissue at the study threshold; however, the threshold was adjusted between three and four $(t=3-4)$ in the uncorrected images, which resulted in showing one cluster that was considered significant at this level, the right inferior temporal gyrus $(\mathrm{t}=3.5$, MNI coordinates: $54,-54,-16)$ (Figure 7).

Males with PD versus females with PD: In PD, females presented with a smaller amount of GM loss compared to males. In females, there was a significant loss in the anterior division of Para hippocampal gyrus not including the amygdala $(\mathrm{t}=5.235$, MNI coordinates: $18,-4,-32)$, the brain stem ( $\mathrm{t}=3.24$, MNI coordinates: $0,-16,-32)$, and a very small cluster in frontal orbital cortex $(t=3.69$, MNI coordinates: $12,6,-20)$ (Figure 8). Males exhibited significant GM loss across the brain in the bilateral crus II of the cerebellum (right: $t=3.66$, MNI coordinates: 20 , -86 , -38; left: $\mathrm{t}=3.54$, MNI coordinates: $-28,-86,-38)$, the bilateral crus I of the cerebellum (right: $\mathrm{t}=4.6, \mathrm{MNI}$ coordinates: $40,-74,-38$; left: $\mathrm{t}=5.5$, $-40,-78,-38)$, the left VIIb of the cerebellum ( $\mathrm{t}=4.24$, MNI coordinates: $-16,-74,-46$ ), the bilateral VI of the cerebellum (right: $t=3.5$, MNI

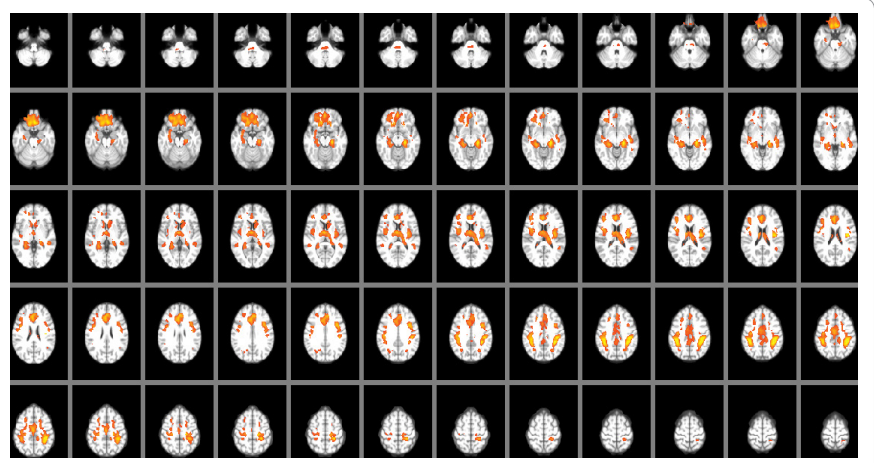

Figure 6: Identified clusters in the VBM comparison between male < female in $\mathrm{HC}$. Increased loss of GM was identified by increasing warmth of colour, representing loss in male $\mathrm{HC}$. (Yellow is warmer)

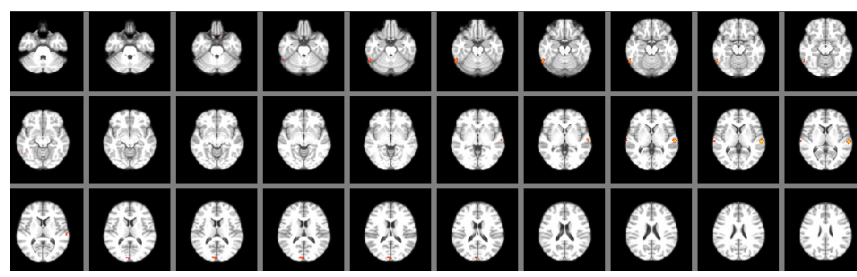

Figure 7: Identified clusters in the VBM comparison between females < males HC. Increased loss of GM was identified by increasing warmth of colour representing a loss in female $\mathrm{HC}$ after adjustment of the threshold from three to four $(t=3-4)$. (Yellow is warmer).

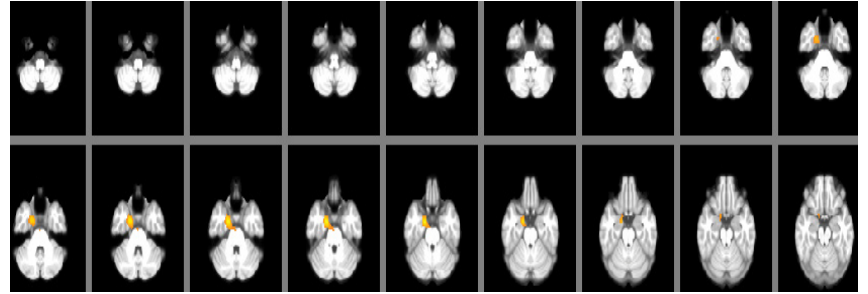

Figure 8: Identified clusters in the VBM comparison between females with PD < males with PD. Increased loss of GM identified by increasing warmth of colour, showing loss in PD females. (Yellow is warmer.)

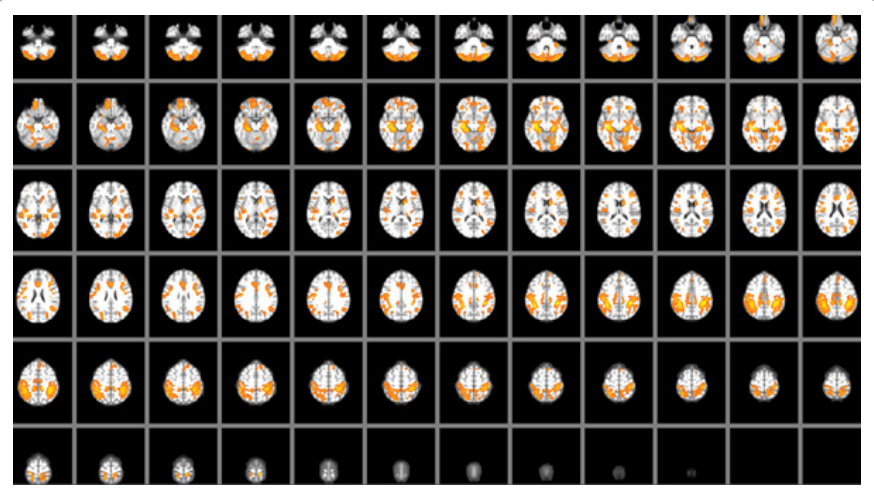

Figure 9: Identified clusters in the VBM comparison between males with PD $<$ females with PD. Increased loss of GM identified by increasing warmth of colour, showing a loss in PD males. (Yellow is warmer.)

coordinates: $10,-74,-24$; left: $\mathrm{t}=3.22$, MNI coordinates: $-12,-68,-24)$, the vermis VI of the cerebellum ( $\mathrm{t}=3.59$, MNI coordinates: $-4,-66,-24)$, the left $\mathrm{V}$ of the cerebellum $(\mathrm{t}=3.74$, MNI coordinates: $-8,-62,-10)$, the bilateral occipital fusiform gyrus (right: $t=5.15$, MNI coordinates: $-22,-78,-10$; left: $t=4$, MNI coordinates: $22,-78,-10)$, the left temporal occipital fusiform ( $\mathrm{t}=4.325$, MNI coordinates: $-28,-56,-8)$, the left occipital cortex ( $t=3.47$, MNI coordinates: $-46,-72,-6)$, the left I-IV of the cerebellum ( $t=3.026$, MNI coordinates: $-4,-50,-6)$, the bilateral hippocampus (right: $\mathrm{t}=6.35$, MNI coordinates: $28,-28,-12$; left: $\mathrm{t}=5.5$, MNI coordinates: $-28,-28,-12$ ), the bilateral fusiform cortex (right: $\mathrm{t}=3.5$, MNI coordinates: $28,-32,-24$; left: $\mathrm{t}=3.06$, MNI coordinates: $-28,-32,-24$ ), the bilateral middle temporal gyrus (right: $t=4.126, \mathrm{MNI}$ coordinates: $48,-34,-2$; left: $t=3.36$, MNI coordinates: $-52,-36,-4)$, the bilateral planum polare (right: $\mathrm{t}=5.116, \mathrm{MNI}$ coordinates: $42,-18,-4$; left: $\mathrm{t}=4.7$, MNI coordinates: $-42,-18,-4$ ), the bilateral amygdala (right: $\mathrm{t}=4.147$, MNI coordinates: $18,-8,-12$; left: $\mathrm{t}=3.44$, MNI coordinates: $-20,-10,-12)$, the right lingual gyrus $(\mathrm{t}=3.46$, MNI coordinates: 16 , $-42,-4$ ), the bilateral insular cortex (right: $\mathrm{t}=3.9$, MNI coordinates: 38 , $-18,0$; left: $t=3.77$, MNI coordinates: $-38,-18,0$ ), the bilateral superior temporal gyrus (right: $t=3.9$, MNI coordinates: $52,-32,0$; left: $t=3.77$, MNI coordinates: $-52,-32,0)$, the left cudate $(\mathrm{t}=4.7$, MNI coordinates: $-14,10,8)$, the bilateral heschl's gyrus (right: $t=3.28$, MNI coordinates: $38,-22,8$; left: $\mathrm{t}=3.22$, MNI coordinates: $-38,-22,8$ ), the left lateral occipital cortex $(t=4.4$, MNI coordinates: $-35,-90,0)$, the bilateral occipital pole (right: $\mathrm{t}=3.16$, MNI coordinates: $10,-98$, 0 ; left: $\mathrm{t}=3.6$, MNI coordinates: $-18,-98,0$ ), the bilateral cingulate gyrus (right: $\mathrm{t}=4.4$, MNI coordinates: 2, 20, 26; left: $t=4.39$, MNI coordinates: $-2,20,26$ ), the bilateral superior parietal lobule (right: $\mathrm{t}=5.3$, MNI coordinates: 26, -52 , 66; left: $t=3.47$, MNI coordinates: $-26,-52,66$ ), the bilateral postcentral gyrus (right: $\mathrm{t}=4.4$, MNI coordinates: $38,-32,66$; left: $\mathrm{t}=5.4$, MNI coordinates: $-38,-32,66$ ), the bilateral supramarginal gyrus (right: $\mathrm{t}=3.8$, MNI coordinates: $56,-32,48$; left: $\mathrm{t}=3.8$, MNI coordinates: -56 , 
$-32,48)$, the bilateral precuneus cortex (right: $\mathrm{t}=4.5$, MNI coordinates: 12, $-42,48$; left: $t=4$, MNI coordinates: $-12,-42,48)$, the bilateral juxta positional lobule (right: $\mathrm{t}=3.3$, MNI coordinates: $8,-12,48$; left: $\mathrm{t}=3.249$, MNI coordinates: $-8,-12,48$ ), the bilateral precentral gyrus (right: $\mathrm{t}=4.28$, MNI coordinates: $38,-22$, 54; left $\mathrm{t}=4$, MNI coordinates: -38 , $-22,54)$, the left frontal pole ( $t=4.16$, MNI coordinates: $6,54,-26)$, the right frontal medial cortex ( $\mathrm{t}=5$, MNI coordinates: $6,44,-26)$, the right subcallosal cortex ( $t=3.3$, MNI coordinates: $6,28,-26)$, the bilateral frontal orbital cortex (right: $\mathrm{t}=5.8$, MNI coordinates: $-38,34,-8$; left: $\mathrm{t}=4.17$, MNI coordinates: $34,32,-8)$, the left thalamus $(\mathrm{t}=3.8$, MNI coordinates: $-18,-28,10)$, the left superior frontal cortex $(t=3.9$, MNI coordinates: $-18,36,52)$, and the right paracingulate gyrus $(\mathrm{t}=3.2, \mathrm{MNI}$ coordinates: 8, 24, 34) (Figure 9 and Table 2).

\begin{tabular}{|c|c|c|c|c|}
\hline \multirow{2}{*}{ Regions affected by tissue loss } & \multicolumn{3}{|c|}{ MNI coordinates } & \multirow{2}{*}{ T score } \\
\hline & $\mathbf{X}$ & $\mathbf{Y}$ & $\mathbf{Z}$ & \\
\hline \multicolumn{5}{|c|}{ Loss of tissues in PD males } \\
\hline Rt. crus II of the cerebellum & 20 & -86 & -38 & 3.66 \\
\hline Lt. crus II of the cerebellum & -28 & -86 & -38 & 3.54 \\
\hline RI. crux I of the cerebellum & 40 & -74 & -38 & 4.6 \\
\hline Lt. crus I of the cerebellum & -40 & -78 & -38 & 5.5 \\
\hline Lt. Vllb of the cerebellum & -16 & -74 & -46 & 4.24 \\
\hline Rt. VI of the cerebellum & 10 & -74 & -24 & 3.5 \\
\hline Lt. VI of the Cerebellum & -12 & -68 & -24 & 3.22 \\
\hline Vormis VI of the cerebellum & -4 & -66 & -24 & 3.59 \\
\hline Lt. $V$ of the cerebellum & -8 & -62 & -10 & 3.74 \\
\hline Rt. occipital fusiform gyros & -22 & -78 & -10 & 5.15 \\
\hline Lt. occipital fusiform gyms & 22 & -78 & -10 & 4 \\
\hline Lt. temporal occipital fusiform & -28 & -56 & -8 & 4.325 \\
\hline Lt. occipital cortex & -46 & -72 & -6 & 3.47 \\
\hline Lt. I-IV of the cerebellum & -4 & -50 & -6 & 3.026 \\
\hline Rt. Hippocampus & 28 & -28 & -12 & 6.35 \\
\hline Lt. hippocampus & -28 & -28 & -12 & 5.5 \\
\hline Rt. fusiform cortex & 28 & -32 & -24 & 3.5 \\
\hline Lt. fusiform cortex & -28 & -32 & -24 & 3.06 \\
\hline Rt. middle temporal gyrus & 48 & -34 & -2 & 4.126 \\
\hline Lt. middle temporal gyrus & -52 & -36 & -4 & 3.36 \\
\hline Rt. planum polare & 42 & -18 & -4 & 5.116 \\
\hline Lt. planum polare & -42 & -18 & -4 & 4.7 \\
\hline Rt. amygdala & 18 & -8 & -12 & 4.147 \\
\hline Lt. amygdala & -20 & -10 & -12 & 3.44 \\
\hline Rt. lingual gyros & 16 & -42 & -4 & 3.46 \\
\hline Rt. insular cortex & 38 & -18 & 0 & 3.9 \\
\hline Lt. insular cortex & -38 & -18 & 0 & 3.77 \\
\hline Rt. superior temporal gyrus & -52 & -32 & 0 & 3.9 \\
\hline Lt. super or temporal gyrus & -52 & -32 & 0 & 3.77 \\
\hline Lt. Cudate & -14 & 10 & 8 & 4.7 \\
\hline Rt. heschi gyrus & 38 & -22 & 8 & 3.28 \\
\hline Lt. heschi gyrus & -38 & -22 & 8 & 3.22 \\
\hline Lt. lateral occipital cortex & -35 & -90 & 0 & 4.4 \\
\hline Rt. occipital pole & 10 & -98 & 0 & 3.16 \\
\hline Lt. occipital pole & -18 & -98 & 0 & 3.6 \\
\hline Rt. cingulate gyrus & 2 & 20 & 26 & 4.4 \\
\hline Lt. cingulate gyrus & 2 & 20 & 26 & 4.39 \\
\hline Rt. superior parietal lobule & 26 & -52 & 66 & 5.3 \\
\hline Lt. superior parietal lobule & -26 & -52 & 66 & 3.47 \\
\hline Rt. postcentral gyrus & 38 & -32 & 66 & 4.4 \\
\hline Lt. postcentral gyrus & -38 & -32 & 46 & 5.4 \\
\hline Rt. Supramarginal gyrus & 56 & -32 & 48 & 3.8 \\
\hline Lt. Supramarginal gyrus & -56 & -32 & 48 & 3.8 \\
\hline Rt. percuneous cortex & 12 & -42 & 40 & 4.5 \\
\hline Lt. percuneous cortex & -12 & -42 & 48 & 4 \\
\hline
\end{tabular}

\begin{tabular}{|c|c|c|c|c|}
\hline Rt. juxta positional lobule & 8 & -12 & 48 & 3.3 \\
\hline Lt. juxta positional lobule & -8 & -12 & 48 & 3.249 \\
\hline Rt. precentral gyrus & 38 & -22 & 54 & 4.28 \\
\hline Lt. precentral gyrus & -38 & -22 & 54 & 4 \\
\hline Lt. frontal pole & 6 & 54 & -26 & 4.16 \\
\hline Rt. frontal medial cortex & 6 & 44 & -26 & 5 \\
\hline Rt. subcallosal cortex & 6 & 28 & -28 & 3.3 \\
\hline Rt. frontal orbital cortex & -38 & 34 & -8 & 5.8 \\
\hline Lt. frontal orbital Cortex & 34 & 32 & -8 & 4.17 \\
\hline LI. thalamus & -18 & -28 & 10 & 3.8 \\
\hline Lt. superior frontal cortex & -18 & 36 & 52 & 3.9 \\
\hline Rt. paracingulate gyrus & 8 & 24 & 34 & 3.2 \\
\hline Loss of tissue In PD females & & \\
\hline Rt. anterior division of Para & 18 & -4 & -32 & 5.235 \\
\hline hippocampal gyrus & 18 & -16 & -32 & 3.24 \\
\hline The brain stern & 0 & -20 & 3.69 \\
\hline Rt. frontal orbital cortex & 12 & 6 & -20 \\
\hline Rt. (nigt); Lt. (Ift). & & & & \\
\hline
\end{tabular}

Note: Rt. (right); Lt. (left).

Table 2: Loss of cortical regions for PD subjects (Males, Females).

\section{Correlation results}

A Pearson correlation was performed to detect any significant correlations between depression scores and the loss of GM tissue for both sexes in PD and HC groups. The MATLAB code to show significant clusters only $(\mathrm{P}<0.05)$. Generally, there were no strong correlations between depression scores and GM in PD and HC. However, they were considered significant because they met the significance level of the study, $\mathrm{P}<0.05$.

Depression correlation to grey matter in Parkinson disease (PD) and healthy control (HC): For correlations between depression and GM changes, without separating both sexes in the PD group, positive correlations were observed in the anterior parts of the cerebellum in bilateral IX (right: $r=0.16$, MNI coordinates: $8,-50,-40$; left: $r=0.17$, MNI coordinates: $-6,-52,-40)$, the vermis IX ( $r=0.17$, MNI coordinates: $0,-52,-34)$, the left VI $(r=0.14$, MNI coordinates: $-30,-50,-26)$, the right I-IV ( $\mathrm{r}=0.17$, MNI coordinates: $4,-50,-22)$, and the left $\mathrm{V}(\mathrm{r}=0.14$, MNI coordinates: $-14,-48,-22)$. Additionally, more regions with positive correlations were presented in the posterior brain stem $(\mathrm{r}=0.16$, MNI coordinates: $6,-38,-26)$, the left temporal fusiform cortex $(r=0.13$, MNI coordinates: $-40,-40,-24)$, the right hippocampus ( $r=0.126$, MNI coordinates: $26,-12,-24)$, the frontal medial cortex $(r=0.19$, MNI coordinates: $-2,38,-18)$, the subcallosal cortex $(r=0.15$, MNI coordinates: $-2,24,-18)$, the bilateral insular cortex (right: $r=0.18$, MNI coordinates: 32, 20, -2; left: $\mathrm{r}=0.12$, MNI coordinates: $-32,20,-2)$, the bilateral thalamus (right: $r=0.15$, MNI coordinates: $18,-20,4$; left: $r=0.13$, MNI coordinates: $-18,-20,4)$, the bilateral central opercular cortex (right: $\mathrm{r}=0.11$, MNI coordinates: $48,-6$, 4; left: $\mathrm{r}=0.13$, MNI coordinates: -48 , $-6,4$ ), the bilateral heschl's gyrus (right: $r=0.136$, MNI coordinates: 44 , $-18,10$; left: $\mathrm{r}=0.11,-44,-18,10$ ), the bilateral intraciliary cortex (right: $r=0.15$, MNI coordinates: $4,-68,12$; left: $r=0.13$, MNI coordinates: -4 , $-68,12$ ), the bilateral precuneus cortex (right: $r=0.17$, MNI coordinates: $8,-62,18$; left: $r=0.16$, MNI coordinates: $-8,-62,18)$, the bilateral anterior cingulate gyrus (right: $r=0.22$, MNI coordinates: $4,-4,40$; left: $r=0.23$, MNI coordinates: $-4,0,40$ ), the bilateral paracingulate gyrus (right: $\mathrm{r}=0.17$, MNI coordinates: 4,46 , 0 ; left: $\mathrm{r}=0.15$, MNI coordinates: $-4,46$, 0 ), the bilateral lingual gyrus (right: $r=0.12, \mathrm{MNI}$ coordinates: $10,-60$, 0 ; left: $r=0.12$, MNI coordinates: $10,-60,0)$, the right middle temporal gyrus ( $\mathrm{r}=0.16$, MNI coordinates: $52,-28,-6)$, the right temporal pole $(\mathrm{r}=0.12$, MNI coordinates: $34,18,-34)$, the bilateral frontal orbital cortex (right: $r=0.19$, MNI coordinates: $38,24,-8$; left: $r=0.129$, MNI coordinates: $-32,28,-2$ ), and the bilateral frontal pole (right: $r=0.139$, 
Citation: Alqarni AA (2017) Structural Brain Sex Differences in Parkinson's Disease: A Voxel-Based Morphometry Study. J Neurol Disord 5: 364 doi:10.4172/2329-6895.1000364

Page 8 of 14

MNI coordinates: 26, 36, -14; left: $r=0.15$, MNI coordinates: $-28,44$, -14). Moreover, negative correlations were observed in the inferior part of the brain stem $(\mathrm{r}=-0.139$, MNI coordinates: $8,-38,-48)$. Various regions of the cerebellum demonstrated negative correlations, such as in the bilateral VIIb (right: $\mathrm{r}=-0.14$, MNI coordinates: $38,-62$, -56; left: $\mathrm{r}=-0.15$, MNI coordinates: $-38,-62,-56$ ), the bilateral crus II (right: $\mathrm{r}=$ -0.146, MNI coordinates: $40,-62,-48$; left: $r=-0.13$, MNI coordinates: $-40,-62,-48)$, the bilateral crus I (right: $r=-0.16$, MNI coordinates: 46 , -62 , -42 ; left: $r=-0.14$, MNI coordinates: $-46,-62,-42)$, the bilateral VIIIa (right: $\mathrm{r}=-0.12$, MNI coordinates: $32,-48,-48$; left: $\mathrm{r}=-0.12$, MNI coordinates: $-32,-48,-48$ ), the bilateral occipital pole (right: $r=$ -0.18 , MNI coordinates: $18,-100,-4$; left: $r=-0.16$, MNI coordinates: $-18,-100,-4)$, the bilateral lateral occipital cortex (right: $r=-0.15$, MNI

\begin{tabular}{|c|c|c|c|c|}
\hline \multirow{2}{*}{ Cortical area of correlations } & \multicolumn{3}{|c|}{ MNI coordinates } & \multirow{2}{*}{ rscore } \\
\hline & $\mathbf{x}$ & $\mathbf{Y}$ & $\mathbf{Z}$ & \\
\hline \multicolumn{5}{|c|}{ Positive } \\
\hline Rt. IX & 8 & -50 & -40 & 0.16 \\
\hline Lt. IX & -6 & -52 & -40 & 0.17 \\
\hline vermis IX & 0 & -52 & -34 & 0.17 \\
\hline left VI & -30 & -50 & -26 & 0.14 \\
\hline right I-IV & 4 & -60 & -22 & 0.17 \\
\hline left $V$ & -14 & -48 & -22 & 0.14 \\
\hline brain stem & 6 & -38 & -26 & 0.16 \\
\hline left temporal fusiform cortex & -40 & -40 & -24 & 0.13 \\
\hline right hippocampus & 26 & -12 & -24 & 0.126 \\
\hline frontal medial cortex & -2 & 38 & -18 & 0.19 \\
\hline subcallosal cortex & 2 & 24 & -18 & 0.15 \\
\hline Rt. Insular cortex & 32 & 20 & -2 & 0.18 \\
\hline Lt. Insular cortex & -32 & 20 & -2 & 0.12 \\
\hline Rt. Thalamus & 18 & -20 & 4 & 0.15 \\
\hline Lt. Thalamus & -18 & -20 & 4 & 0.13 \\
\hline Rt. Central opercular cortex & 48 & -6 & 4 & 0.11 \\
\hline Lt. Central opercular cortex & -48 & -6 & 4 & 0.13 \\
\hline Rt. heschl's gyrus & 44 & -18 & 10 & 0.136 \\
\hline Lt. heschl's gyrus & -44 & -18 & 10 & 0.11 \\
\hline Rt. Intracalcarine cortex & 4 & -68 & 12 & 0.15 \\
\hline Lt. Intracalcarine cortex & -4 & -68 & 12 & 0.13 \\
\hline Rt. precuneous cortex & 8 & -62 & 18 & 0.17 \\
\hline Lt. precuneous cortex & -8 & -62 & 18 & 0.16 \\
\hline Rt. anterior cingulate gyrus & 4 & -4 & 40 & 0.22 \\
\hline Lt. anterior cingulate gyrus & -4 & 0 & 40 & 0.23 \\
\hline Rt. paracingulate gyrus & 4 & 40 & 0 & 0.17 \\
\hline Lt. paracingulate gyrus & -4 & 46 & 0 & 0.15 \\
\hline Rt. lingual gyrus & 10 & -60 & 0 & 0.12 \\
\hline Lt. lingual gyrus & 10 & -60 & 0 & 0.12 \\
\hline right middle temporal gyrus & 52 & -28 & -6 & 0.16 \\
\hline right temporal pole & 34 & 18 & -34 & 0.12 \\
\hline Rt. frontal orbital cortex & 38 & 24 & -8 & 0.19 \\
\hline Lt. frontal orbital cortex & -32 & 28 & -2 & 0.129 \\
\hline Rt. frontal pole & 20 & 30 & -14 & 0.139 \\
\hline Lt. frontal pole & -28 & 44 & -14 & 0.15 \\
\hline \multicolumn{5}{|c|}{ Negative } \\
\hline Rt. VIllb & 38 & -62 & -56 & -0.14 \\
\hline Lt. VIlb & -38 & -62 & -56 & -0.15 \\
\hline Rt. crus II & 40 & -62 & -48 & -0.146 \\
\hline Lt. crus II & -40 & -2 & -40 & -0.13 \\
\hline Rt. crus 1 & 46 & -62 & -42 & -0.16 \\
\hline Lt. crus 1 & -40 & -62 & -42 & -0.14 \\
\hline Rt. Villa & 32 & -48 & -48 & -0.12 \\
\hline Lt. Villa & -32 & -48 & -48 & -0.12 \\
\hline Rt. occipital plate & 18 & -100 & -4 & -0.18 \\
\hline
\end{tabular}

\begin{tabular}{|c|c|c|c|c|}
\hline Lt. occipital plate & -18 & -100 & -4 & -0.16 \\
\hline Rt. lateral occipital cortex & 42 & -82 & -12 & -0.15 \\
\hline Lt. lateral occipital cortex & 42 & -82 & -12 & -0.15 \\
\hline left middle temporal gyrus & -64 & -18 & -12 & -0.15 \\
\hline left inferior temporal gyrus & -58 & -60 & -22 & -0.15 \\
\hline Rt. angular gyrus & 52 & -54 & 26 & -0.15 \\
\hline Lt. angular gyrus & -58 & -58 & 32 & -0.12 \\
\hline Rt. superior parietal lobule & 20 & -50 & 8 & -0.136 \\
\hline Lt. superior parietal lobule & -20 & -50 & 68 & -0.13 \\
\hline Rt. precentral gyrus & 18 & -22 & 68 & -0.16 \\
\hline Lt. precentral gyrus & -18 & -22 & 68 & -0.148 \\
\hline Rt. postcentral gyrus & 22 & -36 & 68 & -0.116 \\
\hline Lt. postcentral gyrus & -22 & -30 & 8 & -0.126 \\
\hline Rt. superior frontal gyrus & 20 & 12 & 66 & -0.12 \\
\hline Lt. superior frontal gyrus & -20 & 12 & 66 & -0.16 \\
\hline Rt. frontal pole & 10 & 52 & 40 & -0.17 \\
\hline Lt. frontal pole & -12 & 50 & 42 & -0.18 \\
\hline left putamen & -24 & 0 & 10 & -0.12 \\
\hline Rt. Superior temporal gyrus & 68 & -34 & 10 & -0.137 \\
\hline Lt. Superior temporal gyrus & -68 & -34 & 10 & -0.15 \\
\hline Not Rt. (right); Lt. (left) & & & & \\
\hline
\end{tabular}

Note: Rt. (right); Lt. (left).

Table 3: The results of the correlation between depression scores and the GM of the PD group (not sex-specific).

coordinates: $42,-82,-12$; left: $r=-0.15$, MNI coordinates: $42,-82,-12)$, the left middle temporal gyrus $(r=-0.15$, MNI coordinates: $-64,-18$, -12 ), the left inferior temporal gyrus ( $\mathrm{r}=-0.15$, MNI coordinates: -58 , $-50,-22$ ), the bilateral angular gyrus (right: $r=-0.15$, MNI coordinates: $52,-54,26$; left: $r=-0.12$, MNI coordinates: $-58,-58,32)$, the bilateral superior parietal lobule (right: $\mathrm{r}=-0.136, \mathrm{MNI}$ coordinates: $20,-50,68$; left: $\mathrm{r}=-0.13$, MNI coordinates: $-20,-50,68)$, the bilateral precentral gyrus (right: $\mathrm{r}=-0.16$, MNI coordinates: $18,-22,68$; left: $\mathrm{r}=-0.148$, MNI coordinates: $-18,-22,68$ ), the bilateral postcentral gyrus (right: $r=$ -0.116 , MNI coordinates: $22,-36,68$; left: $r=-0.126$, MNI coordinates: $-22,-36,68$ ), the bilateral superior frontal gyrus (right: $r=-0.12$, MNI coordinates: $20,12,66$; left: $r=-0.16$, MNI coordinates: $-20,12,66$ ), the bilateral frontal pole (right: $r=-0.17, \mathrm{MNI}$ coordinates: $10,52,40$; left: $\mathrm{r}=-0.18$, MNI coordinates: $-12,50,42)$, the left putamen $(r=-0.12$, MNI coordinates: $-24,0,10)$, and the bilateral superior temporal gyrus (right: $\mathrm{r}=-0.137$, MNI coordinates: $68,-34,10$; left: $\mathrm{r}=-0.15$, MNI coordinates: $-68,-34,10)$ (Table 3 ).

In females of the PD subgroup, weak positive correlations were detected in the right amygdala $(r=0.2$, MNI coordinates: $12,-8,-14)$, the right superior temporal gyrus ( $r=0.228$, MNI coordinates: $60,-18,-2)$, the left precentral gyrus ( $r=0.28$, MNI coordinates: $-8,-20,68)$, the left occipital fusiform gyrus ( $\mathrm{r}=0.2$, MNI coordinates: $-26,-80,-14)$, and the left cuneal cortex $(r=0.217$, MNI coordinates: $-4,-88,26)$. Furthermore, weak negative correlations were found in the right inferior temporal gyrus ( $\mathrm{r}=-0.2$, MNI coordinates: $56,-42,-24)$, the left fusiform gyrus $(\mathrm{r}=-0.2$, MNI coordinates: $-38,-44,-22)$, and the left precuneous cortex $(r=-0.2$, MNI coordinates: $-2,-56,-48$; (Table 4).

Males in the same subgroup showed weak positive correlations in the bilateral precentral gyrus (right: $r=0.18$, MNI coordinates: $2,-18,62$; left: $\mathrm{r}=0.16$, MNI coordinates: $-2,-18,62)$ and the right superior frontal gyrus ( $r=0.24$, MNI coordinates: $18,26,56)$. The negative correlations were detected in PD males in the subcallosal $(r=-0.14$, MNI coordinates: $-2,22,-24)$, the right precuneous cortex $(\mathrm{r}=-0.16$, MNI coordinates: 6 , $-44,48)$, the right hippocampus ( $r=-0.15$, MNI coordinates: $26,-34$, $-4)$, the right amygdala $(r=-0.14$, MNI coordinates: $26,-2,-18)$, and the right lingual gyrus $(r=-0.135$, MNI coordinates: $20,-46,-6)$ (Table 5). 


\begin{tabular}{|c|c|c|c|c|}
\hline \multirow{2}{*}{ Cortical areas of correlations } & \multicolumn{3}{|c|}{ MNI coordinates } & \multirow{2}{*}{ r score } \\
\cline { 2 - 5 } & $\mathrm{X}$ & $\mathrm{Y}$ & $\mathrm{Z}$ & \\
\hline & Positive \\
\hline right amygdala & 12 & -8 & -14 & 0.2 \\
\hline right superior temporal gyrus & 60 & -18 & -2 & 0.228 \\
\hline left precentral gyrus & -8 & -20 & 68 & 0.28 \\
\hline left occipital fusiform gyrus & -26 & -80 & -14 & 0.2 \\
\hline left cuneal cortex & -4 & -88 & 26 & 0.217 \\
\hline Negative & \multicolumn{4}{|l}{} \\
\hline right inferior temporal gyrus & 56 & -42 & -24 & -0.2 \\
\hline left fusiform gyrus & -38 & -44 & -22 & -0.2 \\
\hline left precuneous cortex & -2 & -56 & -48 & -0.2 \\
\hline
\end{tabular}

Table 4: The results of the correlation between depression scores and the GM of females in the PD group.

\begin{tabular}{|c|c|c|c|c|}
\hline \multirow{2}{*}{ Cortical areas of correlations } & \multicolumn{3}{|c|}{ MNI coordinates } & \multirow{2}{*}{ r score } \\
\cline { 2 - 4 } & $\mathbf{X}$ & $\mathbf{Y}$ & $\mathbf{Z}$ & \\
\hline Positive & \multicolumn{4}{|l}{} \\
\hline Rt. precentral gyrus & 2 & -18 & 62 & 0.18 \\
\hline Lt. precentral gyrus & -2 & -18 & 62 & 0.16 \\
\hline Rt. superior frontal gyrus & 18 & 26 & 56 & 0.24 \\
\hline \multicolumn{4}{|c|}{ Negative } \\
\hline Subcallosal & -2 & 22 & -24 & -0.14 \\
\hline Rt. precuneous cortex & 6 & -44 & 48 & -0.16 \\
\hline Rt. hippocampus & 26 & -34 & -4 & -0.15 \\
\hline Rt. amygdala & 26 & -2 & -18 & -0.14 \\
\hline Rt. lingual gyrus & 20 & -46 & -6 & -0.135 \\
\hline
\end{tabular}

Note: Rt. (right); Lt. (left).

Table 5: The results of the correlation between depression scores and the GM of males in the PD group.

In females of the HC subgroup, weak positive correlations were presented in the right precuneus cortex $(\mathrm{r}=0.39$, MNI coordinates: 6 , $-62,14$ ), the bilateral thalamus (right: $r=0.4$, MNI coordinates: $8,-24$, 14; left: $\mathrm{r}=0.3$, MNI coordinates: $-10,-14,2)$, the brain stem $(\mathrm{r}=0.335$, MNI coordinates: $2,-38,-20)$, the right temporal pole $(r=0.28$, MNI coordinates: $26,8,-38)$, the left insular cortex $(r=0.3$, MNI coordinates: $-42,10,-10)$, the right paracingulate gyrus $(\mathrm{r}=0.3$, MNI coordinates: $6,28,36$ ), the bilateral frontal pole (right: $r=0.3$, MNI coordinates: 26 , 52, 4; left: $r=0.3$, MNI coordinates: $-38,40,14)$, the left hippocampus $(\mathrm{r}=0.3$, MNI coordinates: $-26,-10,-28)$, the left temporal fusiform cortex ( $r=0.33$, MNI coordinates: $-34,-8,-44)$, the right IX of the cerebellum ( $\mathrm{r}=0.3$, MNI coordinates: $4,-54,-58)$, and the right lingual gyrus ( $\mathrm{r}=0.33$, MNI coordinates: $10,-62,-4)$. Moreover, weak negative correlations were detected in the bilateral temporal gyrus (right: $r=$ -0.29 , MNI coordinates: $58,-42,-10$; left: $r=-0.4$, MNI coordinates: $-58,-42,-10$ ), the bilateral inferior temporal gyrus (right: $\mathrm{r}=-0.34, \mathrm{MNI}$ coordinates: $52,-56,-22$; left: $r=-0.3$, MNI coordinates: $-58,-42,-22$ ), thebilateral lateral occipital cortex (right: $\mathrm{r}=-0.4$, MNI coordinates: 14 , -76, 50; left: $r=0.4$, MNI coordinates: $-40,-66,46)$, the left crus II of the cerebellum ( $r=-0.33$, MNI coordinates: $-48,-68,-48)$, the bilateral frontal pole (right: $r=-0.3$, MNI coordinates: $8,48,50$; left: $r=-0.27$, MNI coordinates: $-8,48,50)$, the right inferior temporal gyrus $(r=-0.3$, MNI coordinates: $44,-32,-18$ ), the bilateral precentral gyrus (right: $r=$ -0.3 , MNI coordinates: $58,-2,18$; left: $r=-0.39$, MNI coordinates: -58 , $-2,18)$, the right superior frontal gyrus ( $\mathrm{r}=-0.3$, MNI coordinates: 12 , $-2,68)$, and the right VIIb of the cerebellum $(\mathrm{r}=-0.3$, MNI coordinates: $26,-70,-46)$.

On the other hand, males in the same subgroup showed weak positive correlations in the bilateral temporal pole (right: $r=0.4$, MNI coordinates: $30,14,-34$; left: $r=0.2$, MNI coordinates: $-26,14,-34)$, the left middle temporal gyrus ( $\mathrm{r}=0.29$, MNI coordinates: $-58,-20,-12)$, the brain stem ( $r=0.35$, MNI coordinates: $-10,-24,-40)$, the left crus I of the cerebellum ( $r=0.3$, MNI coordinates: $-48,-48,-32)$, the left VI of the cerebellum ( $r=0.28$, MNI coordinates: $-22,-62,-32)$, the right inferior temporal gyrus ( $\mathrm{r}=0.26$, MNI coordinates: $52,-14,-30$ ), the bilateral lateral occipital cortex (right: $r=0.24$, MNI coordinates: $14,-62,64$; left: $\mathrm{r}=0.28$, MNI coordinates: $-32,-68,58)$, the bilateral precuneous cortex (right: $\mathrm{r}=0.3$, MNI coordinates: $10,-52,58$; left: $\mathrm{r}=0.2$, MNI coordinates: $-10,-52,58)$, the left superior parietal lobule $(\mathrm{r}=0.29$, MNI coordinates: $-34,-52,52)$, the right postcentral gyrus ( $\mathrm{r}=0.229$, MNI coordinates: 34 , $-32,53)$, the right supramarginal gyrus $(r=0.238$, MNI coordinates: 52 , $-40,52)$, the left superior frontal gyrus $(\mathrm{r}=0.25$, MNI coordinates: -28 , $16,60)$, the right middle frontal gyrus ( $\mathrm{r}=0.28, \mathrm{MNI}$ coordinates: 30,16 , $56)$, the juxtapositional lobule cortex $(r=0.26$, MNI coordinates: 0,2 , 68 ), the bilateral inferior frontal gyrus (right: $r=0.2$, MNI coordinates: $50,12,20$; left: $r=0.27$, MNI coordinates: $-50,12,20)$, the right thalamus $(\mathrm{r}=0.2$, MNI coordinates: $6,-2,6)$, the left lingual gyrus $(\mathrm{r}=0.29, \mathrm{MNI}$ coordinates: $-28,-46,-6)$, the left superior temporal gyrus $(r=0.29$, MNI coordinates: $-56,-22,-6)$, and the left central opercular cortex $(\mathrm{r}=0.228$, MNI coordinates: $-56,-10,10)$. Additionally, a negative correlation for males of the HC subgroup between depression scores and GM was found in the right precentral gyrus $(r=-0.2$, MNI coordinates: $26,-20,70$ ).

\section{Discussion}

This study has been on the structural brain differences between males and females with PD; A VBM analysis was performed between the PD and HC groups and within each group to identify the major areas that have been affected by PD in both sexes. Moreover, clinical assessments have been investigated to demonstrate the sex differences in the sample of the study. Furthermore, a correlation test was performed to detect any relation between depression and GM changes in $\mathrm{PD}$ and $\mathrm{HC}$.

\section{Demographics characteristics analysis}

This study indicates that the loss of brain tissue affects males and females differently. Additionally, the study indicates that the structural differences in the brain between males and females influence the way $\mathrm{PD}$ is presented clinically by showing more loss brain cortical areas that participate in producing motor and non-motor functions. For instance, males exhibited motor symptoms earlier than females because they were affected by the loss of tissue in areas that have major roles in providing motor control. Moreover, the complexity of the disease relates to the contribution of different factors (e.g., genetical, hormonal, and biochemistries factors) that cannot be covered in neuroimaging studies only. However, studies that investigated the other factors have indicated that females are more protected than males, and the delay of the symptoms presented clinically between males and females is caused independently of higher hazarded occupational factors, the lifestyles of males, and the significantly higher rate of estrogen in females [25-29]. Thus, the results of this study can be shown to emphasize that gender differences in the GM of PD patients exist, males with PD have shown more atrophied cortical areas than females. However, the results from the comparison between males with $\mathrm{PD}$ and $\mathrm{HC}$ males suggest that this atrophy could be due to other factors such as age. Moreover, the degeneration of brain tissue might be the start of the last effects, at least the ones that can be tested clinically, of PD in patients because it was noticed that motor symptoms present after the loss of $60 \%$ of the dopaminergic neurons. Therefore, this study might have the privilege to provide a map of the sex differences in the brain for other researchers who want to investigate dementia or the progression of 
the disease regarding brain morphology, at a mean disease duration of males $6.57 \pm 6.25$ and females $6.8 \pm 6.3$ (months). Furthermore, the study was performed on a large sample of PD and HC subjects, which might have strengthened the study. However, it must be considered that $\mathrm{PD}$ is a heterogeneous disease, implying individual and demographic variations might affect the results of any PD study.

In this study, the sex differences between the PD and HC groups were noticed in several tests. Furthermore, males showed a significant loss of function compared to females in different tests. There were no significant differences between PD and HC subjects in age, as well as between males and females in the PD subgroup. In addition, movement defects were tested by the MDS-UPDRS, and it was expected that the results would be higher in PD than HC because this has been found in previous literature [57]. Moreover, significant differences between both sexes were not found in the current study, and this might be related to the short disease duration at the baseline of the study. However, in a recent study that used a higher disease duration (total PD subjects (years): $7.3 \pm 5.7$; males (years): $7.4 \pm 5.7$; females (years): $7 \pm 5.8$ ), males showed considerably higher movement defect scores than females [65]. To evaluate the disease progression, the Hoehn and Yahr test was performed as a baseline of the disease. At the baseline, the disease duration was short, and this might clarify that no significant result was found between males and females in this study. Additionally, males and females had no difference when tested for daily life activities using the Modified Schwab \& England ADL examination as a baseline; disease duration and the different lifestyles among the subjects might be the reason behind this result.

Furthermore, smelling sense was tested using the University of Pennsylvania Smell Identification Test (UPSIT) to identify any significant differences between $\mathrm{PD}$ and $\mathrm{HC}$ subjects and between males and females within the PD subgroup. At the baseline, PD patients showed significantly higher loss of smell function than HC. Additionally, this result was consistent with a previous study that had studied olfaction in neurodegenerative diseases, including idiopathic PD $[57,66]$. When comparing males and females with PD, significant loss of smelling sense was found more in males than females; this result was consistent with a recent large sample of non-motor symptoms [67]. Moreover, males with PD showed significant loss of GM in the frontal area of the brain compared to females with PD, such as the right frontal medial cortex, the right subcallosal cortex, the bilateral frontal orbital cortex, and in associated areas of the limbic system and temporal lobe, which might aid in the development of this issue [68]. Cognitive defects between PD and HC subjects, as well as between males and females in the PD subgroup, were tested using the Montreal Cognitive Assessment (MoCA). PD patients had significantly higher defects than $\mathrm{HC}$, as expected [57]. Furthermore, loss of cognition function affected males more than females, and this was based on the high level of significance compared to females. The results were consistent with previous literature that had investigated sex differences in $\mathrm{PD}$, suggesting that daily life activities might become affected by the cognitive decline in males more than females $[46,65,67]$. Moreover, autonomic dysfunction was found to be significantly higher in PD patients than in HC. This result was consistent with previous studies [57,67]. In comparing PD males and females, no significant results were found. Interestingly, males in the HC subgroup had more autonomic dysfunction than females, and a difference among the HC group was found, coinciding with a previous study [67].

\section{VBM analysis}

The results of this study showed that different areas of the GM of the brain are significantly atrophied because of PD. The overall differences in PD and HC subjects were concentrated in two separated clusters on the right side of the brain. Those clusters included the right amygdala, the right planum polare, the right temporal pole, and the right Para hippocampal gyrus. The second group of clusters included the right putamen, the right insular cortex, the right central opercular cortex, and the right inferior frontal gyrus (pars opercularis). The results were inconsistent with other studies that have investigated GM using VBM. The inconsistency in the current study might be caused by the increase in the disease duration and the number of subjects included [69-72].

In the PD females versus $\mathrm{HC}$ females, the effects remained on the right side of the brain. Moreover, the areas that were detected contribute to language functions, social emotion functions, autonomic functions, and movement $[73,74]$. The results of the comparison between males in the $\mathrm{PD}$ and $\mathrm{HC}$ subcategory was unexpected. There were no differences in the GM in PD and $\mathrm{HC}$ males at the corrected $\mathrm{T}$ threshold $(\mathrm{t}<3)$ of the study. It is thought that aging affects males more than females $[59,75]$, that might explain the greater loss of cortical areas but show the opposite of normal dimorphism [43]. However, after resetting the threshold from three to four $(t=3-4)$ on the uncorrected images, multiple clusters appeared in the left VI of the cerebellum, the lingual gyrus, the left putamen, the precentral gyrus, and the planum polare for PD male brains, while there was a loss in the subcallosal cortex and the brain-stem for $\mathrm{HC}$ male brains, these areas were found at uncorrected $\mathrm{P}$ value, thus it might not be considered as significant findings.

When comparing $\mathrm{HC}$ males with $\mathrm{HC}$ females, men presented a more significant loss of GM. There was no significant difference in age between both sexes (males: $61 \pm 11.347$; females: $58.7 \pm 11.5$ ). The results of this study indicate that aging affects males more than females. According to Good [59], males had GM reduction in the frontal and temporal regions, and females were affected in the hippocampus and parietal lobe due to normal aging. There were consistencies between the results of this study and the previously mentioned study that looked at the bilateral superior parietal lobule, the insular cortices, the cingulate gyrus, the bilateral planum polare, the thalamus, the hippocampus, the middle temporal gyrus, the right frontal pole, the supramarginal gyrus, the subcallosal cortex, and the left juxta positional lobule cortex. The PD group was divided into males and females, and the VBM results provided maps that showed more GM loss in males than females (males $<$ females). Moreover, the degree of GM tissue loss was expected to be more in males due to the early appearance and the severity of the clinical manifestations than what was seen in the females [25-29]. The loss of the GM tissue affected the areas that are responsible for motor functions, such as the cerebellum, the bilateral superior parietal lobule, the bilateral postcentral gyrus, the bilateral supramarginal gyrus, the bilateral juxta positional lobule, and the bilateral precentral gyrus [76,77]. Also, areas of vision, speech, smell, learning, memory, and cognition were affected in males more than females, including areas in the occipital lobe, the bilateral occipital fusiform gyrus, the left occipital cortex, the left lateral occipital cortex, the bilateral hippocampus, the bilateral amygdala, the bilateral fusiform cortex, the bilateral middle temporal gyrus, the bilateral planum polare, the right lingual gyrus, the bilateral insular cortex, the bilateral superior temporal gyrus, the bilateral heschl's gyrus, the left cudate, the left thalamus, the bilateral occipital pole, the bilateral cingulate gyrus, the bilateral precuneus cortex, the left frontal pole, the right frontal medial cortex, the right subcallosal cortex, the bilateral frontal orbital cortex, the left superior frontal cortex, and the right paracingulate gyrus [78-83]. On the other hand, females presented more atrophy in the anterior division of the Para hippocampal gyrus, the brain stem, and the frontal orbital cortex, 
which involves visuospatial and subperceptual functions, motor skills, recognition of emotions, and decision making [84-86]. In addition, the results of the previous literature did not include any data on gender differences; however, the results of the VBM analysis of the previous study were consistent with the current study.

\section{Correlations analysis}

Pearson correlations were performed to reveal any correlations between depression scores for males and females in the PD and HC subgroups and GM volume, positive correlations indicate increased cortical areas, while negative correlations indicate decreased GM. The correlation between depression and GM volume in the PD subgroup, males and females showed different correlations between depression scores and GM volumes. In females, depression scores were positively correlated with the volumes of the right amygdala, the right superior temporal gyrus, the left cuneal cortex, the left precentral gyrus, and the left occipital fusiform gyrus. The depression scores in females were negatively correlated with the right inferior temporal gyrus, the left fusiform gyrus, and the left precuneus cortex. In males, depression scores were positively correlated with the volumes of the bilateral precentral gyrus and the right superior frontal gyrus, and they were negatively correlated with the right precuneus cortex, the right hippocampus, the right amygdala, and the right lingual gyrus. Additionally, the left precentral gyrus was the only area that showed a similar positive correlation, whereas there were areas that presented an inverse relationship in correlation and location between both sexes, such as the amygdala and the precuneus cortex. In a recent study, a negative correlation between depression and the bilateral hippocampus and the amygdala was identified [87]. The correlation results in the current study indicated this negative correlation between depression and the right side of the hippocampus and the amygdala only in PD males. Thus, this consistency of the results indicates a relation between depression and volume changes in the hippocampus and the amygdala during the progression of the disease which might emphasize the role of the amygdala and the hippocampus in depression. Moreover, the amygdala, especially, has been shown to affect PD [87-89]. Moreover, a positive correlation between the anterior cingulate cortex and the bilateral hippocampus and the amygdala was detected in previous literature, which was not found in the current study. A positive correlation between the bilateral orbitofrontal cortex and GM changes in PD group was also found, while strong negative correlations were found between the right medial temporal gyrus, the right Para hippocampal gyrus, the medial and anterior cingular cortex, and the right cerebellum $[87,90]$. However, the loss of tissue volume in the hippocampus and amygdala is not linked directly to PD [87].

Furthermore, previous literature [87-90] did a comparison of depression scores to cortical areas in the PD group without separating males and females; therefore, a correlation analysis was applied between depression and all PD subjects. A positive correlation was found in the anterior parts of the cerebellum in the bilateral IX, the left VI, the right $\mathrm{I}-\mathrm{IV}$, the left $\mathrm{V}$, and the vermis IX. Also, more positive correlations were found in the posterior part of the brain stem, the left temporal fusiform cortex, the right hippocampus, the subcallosal cortex, the frontal medial cortex, the bilateral insular cortex, the bilateral thalamus, the bilateral central opercular cortex, the bilateral heschl's gyrus, the bilateral intracalcarine cortex, the bilateral precuneus cortex, the bilateral anterior cingulate gyrus, the bilateral paracingulate gyrus, the bilateral lingual gyrus, the right middle temporal gyrus, the right temporal pole, the bilateral frontal orbital cortex, and the bilateral frontal pole. Furthermore, a negative correlation between depression scores and all PD subjects were detected in the inferior part of the brain stem.
Various regions of the cerebellum demonstrated negative correlations in the bilateral VIIb, the bilateral crus II, the bilateral crus I, the bilateral VIIIa, the bilateral occipital pole, the bilateral lateral occipital cortex, the left middle temporal gyrus, the left inferior temporal gyrus, the bilateral angular gyrus, the bilateral superior parietal lobule, the bilateral precentral gyrus, the bilateral postcentral gyrus, the bilateral superior frontal gyrus, the bilateral frontal pole, the left putamen, and the bilateral superior temporal gyrus.

In the control subgroup, males and females presented with different positive and negative correlations in regard to the volume of GM. In females, the depression scores were positively correlated with the right precuneus cortex, the bilateral thalamus, the right temporal pole, the left insular cortex, the right paracingulate gyrus, the bilateral frontal pole, the left hippocampus, the right IX of the cerebellum, the left temporal fusiform cortex, the right lingual gyrus, and the brain stem. The depression scores correlated negatively with the bilateral temporal gyrus, the bilateral inferior temporal gyrus, the bilateral lateral occipital cortex, the left crus II of the cerebellum, the bilateral frontal pole, the bilateral precentral gyrus, the right superior frontal gyrus, and the right VIIb of the cerebellum. In males, the depression scores were positively correlated with the bilateral temporal pole, the left middle temporal gyrus, the brain stem, the left crus I of the cerebellum, the left VI of the cerebellum, the right inferior temporal gyrus, the bilateral lateral occipital cortex, the bilateral precuneus cortex, the left superior parietal lobule, the right postcentral gyrus, the right supramarginal gyrus, the left superior frontal gyrus, the right middle frontal gyrus, the juxta positional lobule cortex, the bilateral inferior frontal gyrus, the right thalamus, the left lingual gyrus, and the left central opercular cortex. The depression scores correlated negatively with the right precentral gyrus. Furthermore, positive and negative correlations between depression and similar cortical areas in one side or bilaterally were found positively in the precuneus cortex, the thalamus, the temporal pole, the lingual gyrus, and the brain stem; these scores correlated negatively in the precentral gyrus. Additionally, areas that exhibited an inverse correlation were identified in the right inferior temporal gyrus and the right superior frontal; this was negative in females and positive in males.

Furthermore, in the previous literature, positive correlations between depression scores and GM volume were found in the bilateral hippocampus, the bilateral putamen, the bilateral nucleus caudatus, the bilateral insular cortex, and the left thalamus, whereas negative correlations were shown in the bilateral medial orbitofrontal cortex [91]. Moreover, there were some consistencies in the results of the current study in regard to depressed HC in another study about the effects of antidepressants on depressed subjects; there were strong positive correlations between depression scores and structural brain images in the left cuneus, the right calcarine gyrus, the left lingual gyrus, and the cerebellum, whereas negative correlations were found in the bilateral middle frontal cortex, the left superior frontal cortex, the bilateral medial frontal cortex, the left inferior frontal cortex, the right anterior midcingulate, the right pregenual anterior cingulate, and the right orbitofrontal cortex [92].

\section{Limitations of the study}

Despite that the original structural data were acquired using a standardized protocol, different scanners and their effects might have affected the identicalness of the image parameters. Moreover, patients who have used antidepressants were not excluded from the study; therefore, the results of the correlation between depression and GM changes might be affected due to the enhancement effects of the 
drugs [45]. Furthermore, although GLM was used to contrast gender differences and age was used as covariate, the study has potential confounds; the data were not corrected to brain volume, education, the onset of the disease in both sexes, and age. Additionally, the lack of spread of depression severity might confound the correlation results.

\section{Conclusion}

This study indicates that the structural differences in Parkinson's disease could influence the clinical manifestations; the structural brain sex differences may suggest sexual brain dimorphism in PD. Moreover, depression correlations to grey matter might confirm the role of the amygdala and the hippocampus in depression-related Parkinson's disease. Furthermore, a collaborative study between neuroimaging and other medical fields should be performed to identify the early effects of PD before brain degeneration starts.

\section{References}

1. Kalia LV, Lang AE (2015) Parkinson's disease. The Lancet 386: 896-912.

2. Wood-Kaczmar A, Gandhi S, Wood NW (2006) Understanding the molecular causes of Parkinson's disease. Trends Mol Med 12: 521-528.

3. Cookson MR, Xiromerisiou G, Singleton A (2005) How genetics research in Parkinson's disease is enhancing understanding of the common idiopathic forms of the disease. Curr Opin Neurol 18: 706-711.

4. Lahiry P, Torkamani A, Schork NJ, Hegele RA (2010) Kinase mutations in human disease: Interpreting genotype-phenotype relationships. Nature Reviews Genetics 11: 60-74.

5. Xiromerisiou G, Dardiotis E, Tsimourtou V, Kountra PM, Paterakis KN, et al. (2010) Genetic basis of Parkinson disease. Neurosurg Focus 28: E7.

6. Jankovic J, Sherer T (2014) The future of research in Parkinson disease. JAMA Neurol 71: 1351-1352.

7. Brown TP, Rumsby PC, Capleton A C, Rushton L, Levy LS (2006) Pesticides and Parkinson's Disease-Is there a link?. Environmental Health Perspectives 114:156-164

8. Ceccatelli S (2013) Mechanisms of neurotoxicity and implications for neurological disorders. J Internal Medicine 273: 426-428.

9. Chinta SJ, Lieu CA, DeMaria M, Laberge R, Campisi J, et al. (2013) Environmental stress, ageing and glial cell senescence: A novel mechanistic link to Parkinson's disease? J Internal Medicine 273: 429-436.

10. Jankovic J (2008) Parkinson's disease: Clinical features and diagnosis. J Neurol Neurosurg Psychiatry 79: 368-376.

11. Pursiainen V, Haapaniemi TH, Korpelainen JT, Sotaniemi KA, Myllylä VV (2007) Sweating in Parkinsonian patients with wearing-off. Mov Disord 22: 828-832.

12. Swinn L, Schrag A, Viswanathan R, Bloem BR, Lees A, et al. (2003) Sweating dysfunction in Parkinson's disease. Mov Disord 18: 1459-1463.

13. Senard JM, Rai S, Lapeyre-Mestre M, Brefel C, Rascol O, et al. (1997) Prevalence of orthostatic hypotension in Parkinson's disease. J Neurol Neurosurg Psychiatry 63: 584-589.

14. Aarsland D, Bronnick K, Ehrt U, De Deyn PP, Tekin S, et al. (2007) Neuropsychiatric symptoms in patients with Parkinson's disease and dementia: Frequency, profile and associated care giver stress. Journal of Neurology, Neurosurgery \& Psychiatry 78: 36-42.

15. Aarsland D, Andersen K, Larsen JP, Lolk A, Nielsen H, et al. (2001) Risk of dementia in Parkinson's disease: A community-based, prospective study. Neurol 56: 730-736.

16. Ondo WG, Dat Vuong K, Khan H, Atassi F, Kwak C, et al. (2001) Daytime sleepiness and other sleep disorders in Parkinson's disease. Neurology 57 1392-1396.

17. Gazewood JD, Richards DR, Clebak K (2013) Parkinson disease: An update Am Fam Physician, 87: 267-273.

18. Postuma RB, Gagnon JF, Montplaisir J (2009) Clinical prediction of Parkinson's disease: Planning for the age of neuroprotection. J Neurol Neurosurg Psychiatry 81: 1008-1013.
19. Rao G (2003) Does this patient have Parkinson disease? JAMA 289: 347.

20. Suchowersky O, Reich S, Perlmutter J, Zesiewicz T, Gronseth G, et al. (2006) Practice parameter: Diagnosis and prognosis of new onset Parkinson disease (an evidence-based review): Report of the quality standards subcommittee of the American academy of Neurology. Neurology 66: 968-975.

21. Hughes AJ, Ben-Shlomo Y, Daniel SE, Lees AJ (1992) What features improve the accuracy of clinical diagnosis in Parkinson's disease: A clinicopathologic study. Neurology 42: 1142-1142.

22. Martin WRW, Wieler M (2003) Treatment of Parkinson's disease. Canadian Journal of Neurological Sciences / Journal Canadien des Sciences Neurologiques 30: S27-S33.

23. Hughes AJ, Daniel SE, Kilford L, Lees AJ (1992) Accuracy of clinical diagnosis of idiopathic Parkinson's disease: a clinico-pathological study of 100 cases. J Neurol Neurosurg Psychiatry 55: 181-184.

24. Wooten GF, (2004) Are men at greater risk for Parkinson's disease than women? J Neurol Neurosurg Psychiatry 75: 637-639.

25. Savica R, Grossardt BR, Bower JH, Ahlskog JE, Rocca WA, (2013) Risk factors for Parkinson's disease may differ in men and women: An exploratory study. Horm Behav 63: 308-314.

26. Saunders-Pullman R, Wang C, Stanley K, Bressman SB, (2011) Diagnosis and referral delay in women with Parkinson's disease. Gend Med 8: 209-217.

27. Haaxma CA, Bloem BR, Borm GF, Oyen WJG, Leenders KL, et al. (2007) Gender differences in Parkinson's disease. J Neurology Neurosurg Psychiatry 78: 819-824.

28. Benedetti MD, Maraganore DM, Bower JH, McDonnell SK, Peterson BJ, et al. (2001) Hysterectomy, menopause, and estrogen use preceding Parkinson's disease: An exploratory case-control study. Movement Disorders 16:830-837.

29. Rocca WA, Bower JH, Maraganore DM, Ahlskog JE, Grossardt BR, et al. (2008) Increased risk of cognitive impairment or dementia in women who underwent oophorectomy before menopause. Neurology 70: 200-209.

30. Pankratz N, Nichols WC, Uniacke SK, Halter C, Rudolph A, et al. (2002) Genome screen to identify susceptibility genes for Parkinson disease in a sample without parkin mutations. Am J Hum Genet 71: 124-135.

31. Benito-León J, Bermejo-Pareja F, Rodríguez J, Molina JA, Gabriel R, et al (2002) Prevalence of PD and other types of parkinsonism in three elderly populations of central Spain. Movement Disorders 18:267-274.

32. Van Den Eeden SK, Tanner C, Berstein A (2003) Incidence of Parkinson's disease: Variation by age gender and race/ethnicity. Am J Epidemiol 157: 1015-1022.

33. Twelves D, Perkins KSM, Counsell C (2003) Systematic review of incidence studies of Parkinson's disease. Mov Disord 18: 19-31.

34. Lyons KE, Hubble JP, Troster Al, Pahwa R, Koller WC (1998) Gender differences in Parkinson's disease. Clin Neuropharmacol 21: 118-121.

35. Zappia M, Annesi G, Nicoletti G, Arabia G, Annesi F, et al. (2005) Sex differences in clinical and genetic determinants of Levodopa peak-dose Dyskinesias in Parkinson disease. Arch Neurol 62: 601

36. Scott B, Borgman A, Engler H, Johnels B, Aquilonius SM (2000) Gender differences in Parkinson's disease symptom profile. Acta Neurol Scand 102 37-43.

37. Martinez-Martin P, Falup Pecurariu C, Odin P, Hilten JJ, Antonini A, et al (2012) Gender-related differences in the burden of non-motor symptoms in Parkinson's disease. J Neurol 259: 1639-1647.

38. McCarthy MM, Arnold AP (2011) Reframing sexual differentiation of the brain Nat Neurosci 14: 677-683.

39. Cosgrove KP, Mazure CM, Staley JK (2007) Evolving knowledge of sex differences in brain structure, function and chemistry. Biol Psychiatry 62: 847-855.

40. Zaidi ZF, (2010) Gender differences in human brain: A review. The Open Anatomy Journal 2: 37-55.

41. Ho KC, Roessmann U, Straumfjord JV, Monroe G (1980) Analysis of brain weight. II. Adult brain weight in relation to body height, weight, and surface area. Archives of Pathology \& Laboratory Medicine 104: 640-645.

42. Goldstein JM, Seidman LJ , Horton NJ , Makris N , Kennedy DN, et al 
(2001) Normal sexual Dimorphism of the adult human brain assessed by in vivo magnetic resonance imaging. Cerebral Cortex 11: 490-497.

43. Ruigrok ANV, Salimi-Khorshidi G, Lai MC, Baron-Cohen S, Lombardo MV, et al. (2014) A meta-analysis of sex differences in human brain structure. Neurosci Biobehav Rev 39: 34-50.

44. Cummings JL (1992) Depression and Parkinson's disease: A review. Am J Psychiatry 149: 443-454.

45. Hamilton JP, Siemer M, Gotlib IH (2008) Amygdala volume in major depressive disorder: A meta-analysis of magnetic resonance imaging studies. Molecular Psychiatry 13: 993-1000.

46. Miller IN, Cronin-Golomb A, (2010) Gender differences in Parkinson's disease: Clinical characteristics and cognition. Mov Disord 25: 2695-2703.

47. Sheline YI, Gado MH, Price JL (1998) Amygdala core nuclei volumes are decreased in recurrent major depression. NeuroReport 9: 2023-2028.

48. Bremner JD, Narayan M, Anderson ER, Staib LH, Miller H, et al. (2000) Hippocampal volume reduction in major depression. American J Psychiatry 157: 115-118.

49. Von Gunten A, Fox NC, Cipolotti L, Ron MA (2000) A volumetric study of Hippocampus and Amygdala in depressed patients with subjective memory problems. J Neuropsychiatry Clin Neurosci 12: 493-498.

50. Frodl T, Meisenzahl EM, Zetzsche T, Born C, Jager M, et al. (2003) Larger amygdala volumes in first depressive episode as compared to recurrent major depression and healthy control subjects. Biological Psychiatry, 53: 338-344.

51. Caetano SC, Hatch JP, Brambilla P, Sassi RB, Nicoletti M, et al. (2004) Anatomical MRI study of hippocampus and amygdala in patients with current and remitted major depression. Psychiatry Research: Neuroimaging, 132: 141 147.

52. Weniger G, Lange C, Irle E (2006) Abnormal size of the amygdala predicts impaired emotional memory in major depressive disorder. J Affect Disord 94: 219-229.

53. Monkul ES, Hatch JP, Nicoletti MA, Spence S, Brambilla P, et al. (2007) Frontolimbic brain structures in suicidal and non-suicidal female patients with major depressive disorder. Mol Psychiatry 12: 360-366.

54. Munn MA, Alexopoulos J, Nishino T, Babb CM, Flake LA, et al. (2007) Amygdala volume analysis in female twins with major depression. Biol Psychiatry 62: $415-422$.

55. Parkinson's progression markers initiative (2015) Available at: http://www,ppmiinfo,org/access-data-specimens/(Accessed: 28 June 2016)

56. Marek K, Jennings D, Lasch S, Siderowf A, Tanner C, et al. (2011) The Parkinson progression marker initiative (PPMI). Progress in Neurobiology 95: 629-635.

57. Jia X, Liang P, Li Y, Shi L, Wang D, et al. (2015) Longitudinal study of grey matter changes in Parkinson disease. Am J Neuroradiology 36: 2219-2226.

58. Douaud G, Smith S, Jenkinson M, Behrens T, Johansen-Berg H, et al. (2007) Anatomically related grey and white matter abnormalities in adolescent-onset schizophrenia. Brain 130: 2375-2386.

59. Good CD, Johnsrude IS, Ashburner J, Henson RNA, Friston KJ, et al. (2001) A Voxel-Based Morphometric study of Ageing in 465 normal adult human brains. Neuro Image 14: 21-36

60. Smith SM, Jenkinson M, Woolrich MW, Beckmann CF, Behrens TEJ, et al. (2004) Advances in functional and structural MR image analysis and implementation as FSL. Neurolmage 23: S208-S219.

61. Andersson JL, Jenkinson M, Smith S (2007) Non-linear registration, aka Spatial normalisation FMRIB technical report TR07JA2. FMRIB Analysis Group of the University of Oxford 2.

62. Ségonne F, Dale AM, Busa E, Glessner M, Salat D, et al. (2004) A hybrid approach to the skull stripping problem in MRI. Neurolmage 22: 1060-1075.

63. MATLAB and Statistics Toolbox Release R2016a The Math Works Inc, Natick Massachusetts United States.

64. Winkler AM, Ridgway GR, Webster MA, Smith SM, Nichols TE (2014) Permutation inference for the general linear model. Neurolmage 92: 381-397.

65. Lubomski M, Rushworth LR, Lee W, Bertram KL, Williams DR (2014) Sex differences in Parkinson's disease. J Clinical Neuroscience 21: 1503-1506.
66. Hawkes C (2003) Olfaction in neurodegenerative disorder. Movement Disorder 18: $364-372$

67. Liu R, Umbach DM, Peddada SD, Xu Z, Troster Al, et al. (2015) Potential sex differences in nonmotor symptoms in early drug-naive Parkinson disease. Neurology 84: 2107-2115.

68. Sanders R D, Gillig PM (2009) Cranial Nerve I: Olfaction. Psychiatry (Edgmont) 6: 30-35.

69. Summerfield C, Junqué C, Tolosa E, Salgado-Pineda P, Gómez-Ansón B, et al. (2005) Structural brain changes in Parkinson disease with dementia. Arch Neurol 62: 281.

70. Beyer MK, Janvin CC, Larsen JP, Aarsland D (2006) A magnetic resonance imaging study of patients with Parkinson's disease with mild cognitive impairment and dementia using voxel-based morphometry. J Neurol Neurosurg Psychiatry 78: 254-259.

71. Pereira JB, Ibarretxe-Bilbao N, Marti MJ, Compta Y, Junqué C, et al. (2011) Assessment of cortical degeneration in patients with Parkinson's disease by voxel-based morphometry cortical folding and cortical thickness. Hum Brain Mapp 33: 2521-2534.

72. Pan PL, Shi HC, Zhong JG, Xiao PR, Shen Y, et al. (2012) Gray matter atrophy in Parkinson's disease with dementia: Evidence from meta-analysis of voxelbased morphometry studies. Neurol Sci 34: 613-619.

73. Rizzolatti G, Fogassi L, Gallese V (2002) Motor and cognitive functions of the ventral premotor cortex. Curr Opin Neurobiol 12: 149-154.

74. Nieuwenhuys R (2011) The insular cortex: A review. Prog Brain Res 195: 123 163.

75. Coffey CE, Lucke JF, Saxton JA, Ratcliff G, Unitas LJ, et al. (1998) Sex differences in brain aging. Archives of Neurology 55: 169-179.

76. Morton SM, Bastian AJ (2004) Cerebellar control of balance and locomotion The Neuroscientist 10: 247-259.

77. Chouinard PA, Paus T (2006) The primary motor and premotor areas of the human cerebral cortex. The neuroscientist 12: 143-152.

78. Ramírez-RuizB, Martí MJ, Tolosa E, Bartrés-Faz D, Summerfield C, et al. (2005) Longitudinal evaluation of cerebral morphological changes in Parkinson's disease with and without dementia. J Neurol 252: 1345-1352.

79. Nagano-Saito A, Washimi Y, Arahata Y, Kachi T, Lerch JP, et al. (2005) Cerebra atrophy and its relation to cognitive impairment in Parkinson disease. Neurology 64: 224-229.

80. Carlesimo GA, Piras F, Assogna F, Pontieri FE, Caltagirone C, et al. (2012) Hippocampal abnormalities and memory deficits in Parkinson disease: A multimodal imaging study. Neurol 78: 1939-1945

81. O'Callaghan C, Moustafa AA, De Wit S, Shine JM, Robbins TW, et al. (2013) Fronto-striatal gray matter contributions to discrimination learning in Parkinson's disease. Front Comput Neurosci 7: 180

82. Arnold C, Gehrig J, Gispert S, Seifried C, Kell CA (2014) Pathomechanisms and compensatory efforts related to Parkinsonian speech. Neurolmage: Clinical 4:82-97.

83. Zhao Y, Zheng X, Wang Q, Xu J, Xu X, et al. (2014) Altered activation in visual cortex: Unusual functional magnetic resonance imaging finding in early Parkinson's disease. J Int Med Res 42: 503-515.

84. Ibarretxe-Bilbao N, Junque C, Tolosa E, Marti MJ, Valldeoriola F, et al. (2009) Neuroanatomical correlates of impaired decision-making and facial emotion recognition in early Parkinson's disease. European J Neuroscience 30: 11621171.

85. Jubault T, Brambati SM, Degroot C, Kullmann B, Strafella AP, et al. (2009) Regional brain stem atrophy in Idiopathic Parkinson's disease detected by anatomical MRI. PLoS ONE 4: e8247.

86. Ibarretxe-Bilbao N, Junque C, Marti MJ, Tolosa E (2011) Brain structural MR correlates of cognitive dysfunctions in Parkinson's disease. J Neurological Sciences 310: 70-74.

87. Van Mierlo TJ, Chung C, Foncke EM, Berendse HW, Van den Heuvel OA (2015) Depressive symptoms in Parkinson's disease is related to decreased hippocampus and amygdala volume. Mov Disord 30: 245-252.

88. Burke J, McQuoid DR, Payne ME, Steffens DC, Krishnan RR, et al. (2011) Amygdala volume in late-life depression. Am J Geriatr Psychiatry 19: 771-776. 
Citation: Alqarni AA (2017) Structural Brain Sex Differences in Parkinson's Disease: A Voxel-Based Morphometry Study. J Neurol Disord 5: 364. doi:10.4172/2329-6895.1000364

Page 14 of 14

89. Surdhar I, Gee M, Bouchard T, Coupland N, Malykhin N, et al. (2012) Intact limbicprefrontal connections and reduced amygdala volumes in Parkinson's disease with mild depressive symptoms. Parkinsonism Relat Disord 18: 809-813.

90. Feldmann A, Illes Z, Kosztolanyi P, Illes E, Mike A, et al. (2008) Morphometric changes of gray matter in Parkinson's disease with depression: A voxel-based morphometry study. Movement Disorders 23: 42-46.
91. Egger K, Schocke M, Weiss E, Auffinger S, Esterhammer R, et al. (2008) Pattern of brain atrophy in elderly patients with depression revealed by voxelbased morphometry. Psychiatry Research: Neuroimaging 164: 237-244.

92. Chen CH, Ridler K, Suckling J, Williams S, Fu CHY, et al. (2007) Brain imaging correlates of depressive symptom severity and predictors of symptom improvement after antidepressant treatment. Biological Psychiatry 62: 407-414. 